\title{
Endogenous Tuberculous Endophthalmitis and Panophthalmitis: A Systematic Review of Case Reports and Case Series
}

This article was published in the following Dove Press journal: Clinical Ophthalmology

\author{
Fares Antaki (D) ${ }^{1,2, *}$ \\ Simon Javidi (iD) ${ }^{1,2, *}$ \\ Samir Touma' \\ Marie-Josée Aubin (D) ${ }^{1-3}$ \\ 'Centre Universitaire d'Ophtalmologie \\ (CUO), Hôpital Maisonneuve-Rosemont, \\ CIUSSS de l'Est-de-L'île-de-Montréal, \\ Montreal, Quebec, Canada; ${ }^{2}$ Department \\ of Ophthalmology, Université de \\ Montréal, Montreal, Quebec, Canada; \\ ${ }^{3}$ Department of Social and Preventive \\ Medicine, School of Public Health, \\ Université de Montréal, Montreal, \\ Quebec, Canada \\ *These authors contributed equally to \\ this work
}

Purpose: To systematically review the characteristics of patients with endogenous tuberculous (TB) endophthalmitis and panophthalmitis in an effort to help clinicians with diagnosis and treatment.

Patients and Methods: We conducted a systematic literature search in MEDLINE/ PubMed, EMBASE and Web of Science from inception to August 2020. References and abstracts were screened independently by two authors. Included studies were case reports and case series reporting endogenous TB endophthalmitis and panophthalmitis secondary to Mycobacterium tuberculosis complex (MTBC). Available-case analysis was employed to handle missing data.

Results: A total of 1343 articles were found using the search strategy. Following abstract screening, 51 articles were selected for full text-review, from which 26 were deemed eligible for inclusion in the study. Forty-four cases from 26 articles were included in the quantitative analysis. The median age of presentation was 29.5 (range: 1 to 81), and 11/44 patients $(25.0 \%)$ were pediatric. Immunosuppression was seen in 9/36 cases $(25.0 \%)$. Most patients $(24 / 38,63.2 \%)$ had no prior history of tuberculosis. Systemic symptoms were absent in half of the patients $(16 / 32,50.0 \%)$. Visual acuity was poor, with $23 / 27$ cases $(85.2 \%)$ being 20 / 200 or worse at presentation. Poor organ and visual outcomes were reported: 36/43 cases $(83.7 \%)$ resulted in enucleation/evisceration or exenteration. Intraocular tumors were suspected in 5/34 cases (14.7\%). Pulmonary tuberculosis was seen in $15 / 35$ cases $(42.8 \%)$, and miliary tuberculosis was seen in $7 / 35$ cases $(20.0 \%)$. The earliest source of TB diagnosis was through histopathologic specimen after eye removal in 32/44 cases $(72.7 \%)$, vitreous specimen in $6 / 44$ cases $(13.6 \%)$ and aqueous specimen in $3 / 44$ cases $(6.8 \%)$.

Conclusion: TB endophthalmitis is a rare and sight-threatening manifestation of ocular tuberculosis. It can occur in apparently healthy individuals and can mimic intraocular tumors and other infectious etiologies. Diagnosis remains a significant challenge, which, often delayed, leads to profound visual loss. Early detection and treatment of intraocular tuberculosis may be associated with better ocular and systemic outcomes.

Keywords: systematic review, tuberculosis, endophthalmitis, panophthalmitis, panuveitis

\section{Introduction}

Tuberculosis is a major global health issue with an important burden of disease, leading to significant mortality and morbidity worldwide. ${ }^{1}$ Despite progress in detection and treatment of tuberculosis and a decline in the mortality over the last decade, more than 1.3 million individuals died from this disease in 2017. It is estimated that around a third of the world's population is infected with Mycobacterium tuberculosis. ${ }^{2}$ The
Correspondence: Fares Antaki

Department of Ophthalmology, Hôpital Maisonneuve-Rosemont, CIUSSS de l'Estde-L'île-de-Montréal, 54I5 Assumption Blvd, Montreal, QC HIT 2M4, Canada Email fares.antaki@umontreal.ca 
prevalence and incidence of tuberculosis in the United States is significantly lower than global numbers: in 2018, there were 9,000 new cases of active tuberculosis and 515 deaths due to the disease. ${ }^{3}$

Tuberculosis is an airborne bacterial infection caused by Mycobacterium tuberculosis complex (MTBC) which typically affects the lungs. Tuberculosis is most commonly caused by Mycobacterium tuberculosis but it can also be secondary to Mycobacterium bovis, which accounts for less than $1.6 \%$ of cases in North America. ${ }^{4,5}$ In contrast, recent studies from Mexico noted a high prevalence and a rising trend of tuberculosis caused by M. bovis, representing up to $28.4 \%$ of cases in one study. ${ }^{6,7}$

Live tubercle bacilli have the capacity of propagating through hematogenous and lymphatic spread to seed in lymph nodes, bones and kidneys. ${ }^{8}$ Multiple risk factors have been identified for extrapulmonary tuberculosis such as female gender, age above forty and patients with concomitant human immunodeficiency virus (HIV). ${ }^{8}$ Intraocular tuberculosis is a rare form of extrapulmonary tuberculosis and is more common in patients with HIV. ${ }^{8}$

Intraocular tuberculosis accounts for $2 \%$ to $18 \%$ of extrapulmonary tuberculous (TB) manifestations. ${ }^{9}$ Tuberculosis has an increased propensity for tissues with high oxygen regional tension such as the choroid and the ciliary body. Uveitis represents the most common form of intraocular tuberculosis and has different presentations. Anterior uveitis is typically granulomatous and occurs in $12-36 \%$ of cases. ${ }^{9}$ Posterior uveitis accounts for $11-20 \%$ of cases of TB uveitis whereas panuveitis is more common, with $35-42 \%$ of cases. ${ }^{9}$ Posterior segment manifestations also include multifocal choroiditis, ${ }^{10-13}$ serpiginous-like choroiditis, ${ }^{14}$ chorioretinitis $^{11}$ and tuberculomas. ${ }^{15}$

Endophthalmitis is a severe presentation of intraocular tuberculosis that progresses rapidly and that can lead to intraocular tissue destruction as well as globe perforation when the cornea or the sclera are involved (panophthalmitis). Given the scarcity of literature on the topic, we performed a systematic review to evaluate patient characteristics, clinical features, diagnostic tests and outcomes of patients with TB endophthalmitis and panophthalmitis.

\section{Methods}

\section{Definitions and Eligibility Criteria}

Case reports and case series reporting original data of endogenous TB endophthalmitis and panophthalmitis were included. Endogenous TB endophthalmitis was defined as purulent intraocular inflammation due to hematogenous spread of MTBC, with rapid progression. Panophthalmitis was defined as severe acute suppurative intraocular inflammation with extension to the cornea or sclera, secondary to endogenous spread of MTBC. A priori, to avoid excluding older studies, our definition of MTBC infection did not require a confirmed mycobacterial culture or positive polymerase chain reaction (PCR). Rather, all cases that were reported as endogenous "TB endophthalmitis" or "panophthalmitis" by the original authors were included. Cases, that were not explicitly labeled as "endophthalmitis" or "panophthalmitis" by the original authors, meeting our definition were also included, after careful review of each case. Cases of multifocal choroiditis, serpiginous choroiditis, chorioretinitis and tuberculomas without an associated rapidly progressive purulent panuveitis were excluded. Studies reporting post-operative and post-traumatic endophthalmitis and cases of non-tuberculous mycobacteria were excluded. We also excluded all pathological studies not including enough clinical information, studies that contained nonoriginal data and those with no full-text available. Studies in languages other than English that could not be translated by the authors were also excluded.

\section{Search Strategy}

The systematic review was conducted in adherence with the PRISMA guidelines. ${ }^{16}$ The databases were systematically searched from inception to August 1st 2020. References of relevant studies were also screened using the snowballing method.

The MEDLINE/PubMed search was conducted using the following terms: (Endophthalmitis [mh] OR panuveitis [mh] OR endophthalmitis [tiab] OR panophthalmitis [tiab]) AND (Tuberculosis [mh] OR Mycobacterium tuberculosis [mh] OR Mycobacterium bovis [mh] OR Tuberculosis [tiab] OR bovis [tiab] OR tuberc*).

The EMBASE search was performed using the following headings: (exp endophthalmitis/OR exp panophthalmitis/OR panuveitis.mp. OR endophthalmitis.mp. OR panophthalmitis.mp.) AND (exp Mycobacterium tuberculosis/OR exp Mycobacterium bovis/OR exp tuberculosis/ OR tuberculosis.mp. OR bovis.mp. OR tubercul*.mp.) NOT ("animal"/exp NOT "human"/exp).

The Web of Science search strategy was the following: $\mathrm{TS}=($ Endophthalmitis OR panophthalmitis OR panuveitis) 
AND TS $=($ Mycobacterium tuberculosis OR Mycobacterium bovis OR tuberculosis OR bovis OR tuberc*).

\section{Screening Process}

References were imported into Covidence systematic review software (Veritas Health Innovation, Melbourne, Australia. Available at www.covidence.org) for screening and eligibility assessment. FA and SJ independently screened the titles and abstracts obtained using the abovementioned search strategy. Additional records identified through snowballing of reference lists were also screened. Full texts of studies that met the inclusion criteria or that needed further screening were examined independently. Disagreements were resolved through consultation and a final decision was taken by the senior author (MJA) when needed. When studies were excluded, reasons were clearly outlined.

\section{Data Collection}

The following information was extracted from the included studies: authors' names, year of publication, type of study (case series or case report), patient's demographics (age and sex), past medical history (including history of tuberculosis), ocular and systemic symptoms on presentation, ocular examination findings on presentation, working diagnosis as reported by the authors, clinical evolution including medical and surgical treatments received, diagnostic tests including chest $\mathrm{x}$-ray (CXR), chest computed tomography (CT), tuberculin skin test (TST) and interferon-gamma release assays (IGRA) results, final diagnosis, source and method of tuberculosis identification, and ocular morbidity and mortality outcomes. Quantitative data was presented by number of cases and occurrences with percentages. To handle missing data, a pairwise deletion approach (available-case analysis) was employed which allowed us to use the case when analyzing other non-missing variables. ${ }^{17}$

\section{Results}

\section{Study Selection and Characteristics}

Figure 1 shows the PRISMA flow chart. The electronic search provided 1343 articles. Two hundred and seventysix duplicates were identified by the systematic review software. Following title and abstract screening of 1067 studies, 1016 studies were excluded. Full-text eligibility assessment of 51 articles was performed. After exclusion of 25 full-text articles, 21 studies were deemed eligible and included in the study. The study characteristics are summarized in Table 1. Detailed case-by-case descriptions are available in Tables 2 and 3. There were 7 case series and 19 case reports. The earliest publication was from 1958 and the latest from 2019. There were 104 cases in the 26 studies. Only 44 cases were deemed eligible after careful case-by-case assessment of the case series.

\section{Demographic Data and Past Medical History}

The articles yielded 44 patients of whom 11 were pediatric (25.0\%). The median age was 29.5 (interquartile range, IQR $=27$ ) and ages ranged from 1 to 81 years. There were 26 males (59.1\%) and 18 females (40.9\%). Table 4 summarizes the demographic data and past medical histories. Among the 36 patients where a past medical history was detailed, 27 were immunocompetent (75.0\%) and 9 were immunocompromised (25.0\%). Patients who were empirically started on systemic corticosteroids after the beginning of ocular symptoms were not considered immunosuppressed based on their past medical history. Six patients had HIV with low CD4 count (range: $34-263 \mathrm{cell} / \mathrm{mm}^{3}$ ). ${ }^{18-21}$ One was known for Waldenström's macroglobulinemia (WM) and rituximabassociated hypogammaglobulinemia. ${ }^{22}$ Two patients were on immunosuppressive therapy for systemic sarcoidosis and systemic lupus erythematosus. ${ }^{23,24}$ Most patients (24/ 38 ) had no prior history of systemic TB (63.2\%), making the ocular presentation the first sign of the disease. Only $6 /$ 37 patients (16.2\%) had active TB infections prior to ocular presentation. Bacille Calmette-Guérin (BCG) vaccination status was not reported in any of the cases.

\section{Initial Presentation and Visual Acuity}

TB endophthalmitis and panophthalmitis had varied presentations in the available reports. Contralateral eye findings were reported in 34/44 cases. All cases of TB endophthalmitis or panophthalmitis involved a single eye per patient. However, in $4 / 34$ cases (12\%), the contralateral eye was affected to a lesser degree (eg, chorioretinal lesion) and/or was not diagnosed as "endophthalmitis" or "panophthalmitis" by the original authors, thereby not meeting our eligibility criteria and not included in the analysis. $^{23,25-27}$ There were no cases of bilateral TB endophthalmitis or panophthalmitis. Table 5 summarizes the clinical characteristics and working diagnoses on presentation. The principal presenting symptoms were decreased vision $(28 / 31,90.3 \%)$, ocular pain or headache 


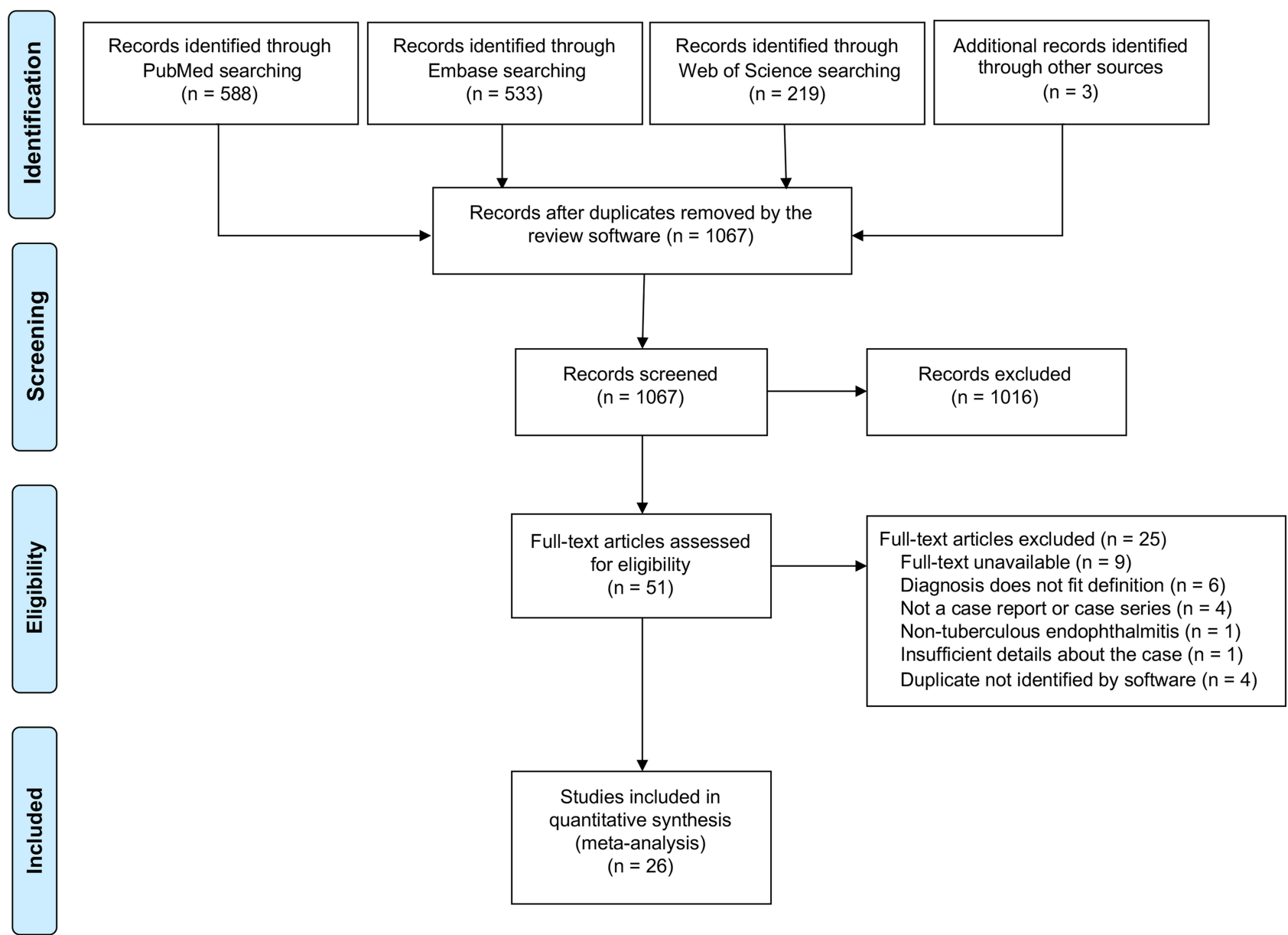

Figure I Preferred Reporting Items for Systematic Reviews and Meta-Analyses (PRISMA) flow diagram. ${ }^{16}$

$(18 / 31,58.1 \%)$ and redness $(10 / 31,32.3 \%)$. Other symptoms included abnormal protrusion of the eye $(2 / 31$, $6.5 \%)$, floaters $(2 / 31,6.5 \%)$ and epibulbar mass $(2 / 31$, $6.5 \%$ ). Symptom duration varied and a significant number of patients had symptoms for 1 to 3 months prior to presentation (15/30,50.0\%). Only 5/30 patients consulted within a week of symptom presentation (16.7\%). Purulent intraocular inflammation was present in all cases and was a defining criterion for inclusion in this study. Other examination findings on initial presentation and during follow-up included: impaired ocular motility in $7 / 44$ cases $(15.9 \%)$, extraocular mass or scleral abscess in $7 /$ 44 cases (15.9\%), high intraocular pressure or glaucoma in $6 / 44$ cases $(13.6 \%)$, spontaneous perforation in 3/44 cases $(6.8 \%)$ and necrotizing retinitis in $2 / 44$ cases $(4.5 \%)$. Systemic symptoms like fever, chills or cough prior to presenting to the ophthalmology clinic were absent in half of the patients $(50.0 \%)$. Visual acuities on presentation were generally poor: $23 / 27(85.2 \%)$ had 20/200 vision or worse. Almost half of the patients (12/27, 44.4\%) had no light perception (NLP) vision.

\section{Working Diagnosis}

In $18 / 34$ cases (52.9\%), endophthalmitis or panophthalmitis of unknown etiology (pending investigations) was considered as a working diagnosis. Non-tuberculous infectious and inflammatory etiologies were considered in multiple cases: acute retinal necrosis (ARN) (2 cases), ${ }^{22,25}$ syphilitic uveitis $(2 \text { cases })^{18,28}$ and sarcoid uveitis ( 1 case) ${ }^{29}$ Retinoblastoma was suspected in $4 / 34$ cases (11.8\%), representing $36.4 \%$ of all pediatric cases. Metastatic carcinoma was suspected in one case in an adult patient. ${ }^{28,30-33}$ Only 2 cases (5.9\%) reported a TB etiology as a working diagnosis, in a patient with known systemic tuberculosis and "contralateral choroidal masses", and in another with a positive CXR obtained at presentation. ${ }^{23,34}$ Among the 3 other patients with contralateral ocular findings, endogenous endophthalmitis of unknown etiology was suspected in 2 cases without 
Table I Published Cases of Tuberculous Endogenous Endophthalmitis and Panophthalmitis in the Literature

\begin{tabular}{|c|c|c|c|c|c|c|}
\hline No. & Author & Year & Source & Type of Study & Cases & Eligible Cases \\
\hline I & Dvorak-Theobald ${ }^{37}$ & 1958 & Search strategy & Case report & I & I \\
\hline 2 & Darrell $^{36}$ & 1967 & Search strategy & Case report & I & I \\
\hline 3 & McMoli et $\mathrm{al}^{32}$ & 1978 & Search strategy & Case report & 1 & I \\
\hline 4 & Manthey et $\mathrm{al}^{56}$ & 1982 & Search strategy & Case report & I & I \\
\hline 5 & $\mathrm{Ni}$ et $\mathrm{al}^{28}$ & 1982 & Search strategy & Case series & 6 & 3 \\
\hline 6 & Menezo et $\mathrm{al}^{18}$ & 1987 & Search strategy & Case report & 1 & I \\
\hline 7 & Regillo et $\mathrm{al}^{29}$ & 1991 & Snowballing & Case report & I & I \\
\hline 8 & Anders and Wollensak ${ }^{24}$ & 1995 & Search strategy & Case report & I & I \\
\hline 9 & Biswas et $\mathrm{al}^{23}$ & 1995 & Search strategy & Case series & 5 & 2 \\
\hline 10 & Raina et $\mathrm{al}^{30}$ & 2000 & Search strategy & Case report & I & 1 \\
\hline II & Sheu et a ${ }^{57}$ & 2001 & Search strategy & Case series & 5 & 2 \\
\hline 12 & Grosse et $\mathrm{al}^{33}$ & 2002 & Snowballing & Case report & I & I \\
\hline 13 & Sen et $\mathrm{al}^{31}$ & 2003 & Search strategy & Case report & 1 & 1 \\
\hline 14 & Chawla et a ${ }^{58}$ & 2004 & Search strategy & Case report & I & I \\
\hline 15 & Demirci et $\mathrm{al}^{53}$ & 2004 & Search strategy & Case series & 5 & 2 \\
\hline 16 & Babu et al ${ }^{19}$ & 2006 & Search strategy & Case series & 15 & 3 \\
\hline 17 & Wadhwani et $\mathrm{al}^{34}$ & 2011 & Search strategy & Case report & 1 & 1 \\
\hline 18 & Wroblewski et $\mathrm{al}^{59}$ & 2011 & Search strategy & Case series & 42 & 8 \\
\hline 19 & Sengupta et $\mathrm{al}^{26}$ & 2013 & Search strategy & Case report & I & 1 \\
\hline 20 & Hase et $\mathrm{al}^{35}$ & 2015 & Search strategy & Case report & I & I \\
\hline 21 & Srichatrapimuk et $\mathrm{al}^{21}$ & 2016 & Search strategy & Case report & 1 & 1 \\
\hline 22 & Agarwal et $\mathrm{al}^{60}$ & 2017 & Online search & Case report & I & I \\
\hline 23 & Boonsopon et $\mathrm{al}^{20}$ & 2017 & Search strategy & Case report & I & 1 \\
\hline 24 & Rishi et al ${ }^{25}$ & 2018 & Search strategy & Case series & 5 & 5 \\
\hline 25 & Antaki et $\mathrm{al}^{22}$ & 2019 & Search strategy & Case report & 1 & 1 \\
\hline 26 & Yaghoubi et $\mathrm{al}^{27}$ & 2019 & Search strategy & Case report & 1 & I \\
\hline \multicolumn{6}{|c|}{ Total number of eligible cases } & 44 \\
\hline
\end{tabular}

a specific suspicion for TB. ${ }^{25,27}$ The last case, published by Sengupta et al, did not report a working diagnosis. ${ }^{26}$

\section{Treatments and Investigations Prior to TB Diagnosis}

Table 6 summarizes the clinical course, interventions and outcomes. Before a tuberculosis diagnosis was confirmed, most patients received systemic antibiotics, corticosteroids or a combination of both. Corticosteroids alone were used in $7 / 21$ cases (33.3\%) and in combination with antibiotics in $3 / 21$ cases $(14.3 \%)$. Antibiotics alone were prescribed in $5 / 21$ cases $(23.8 \%)$. Antivirals were used in $2 / 21$ cases $(9.5 \%)$ of suspected ARN, in conjunction with corticosteroids ( 1 case $)^{25}$ or antibiotics ( 1 case). ${ }^{22}$ Radiation therapy was used in one suspected case of retinoblastoma. ${ }^{32}$ When chest imaging was obtained, CXR or CT scan were abnormal in 19/27 cases (70.4\%) and normal in 8/27 (29.6\%). Among the 19 cases with abnormal chest studies, 13/19 (68.4\%) had an abnormal CXR as reported by the authors.
Abnormal findings included lymphadenopathy, "lesions", "active infection", pleural effusions and consolidations. Cases with reported CXR findings (eg, calcified granuloma) that were not deemed compatible with an active infection by the original authors were considered normal. In 4/19 cases, despite a normal initial CXR, miliary mottling was apparent on repeat CXR ( 1 case) and on subsequent $\mathrm{CT}$ scan ( 3 cases). ${ }^{22,26,35}$ In 2 cases, miliary mottling was apparent on initial CXR. ${ }^{25}$ When a TST (Mantoux test) was reported, $12 / 20(60.0 \%)$ were positive, $6 / 20$ $(30.0 \%)$ were negative and $2 / 20(10.0 \%)$ converted from negative to positive during the follow-up period. ${ }^{18,36}$ Among the 9 immunocompromised individuals, a TST result was available in 4 cases. It was positive in 2 cases, negative in 1 case and converted in 1 case. IGRA test results were only reported in 4 cases by Rishi et al. ${ }^{25}$ Among those 4 cases, 2 patients had a negative TST and a negative IGRA. One patient had a negative TST but a positive IGRA. The last patient had a positive TST and a positive IGRA. 


\begin{tabular}{|c|c|c|c|c|c|c|c|c|c|c|}
\hline 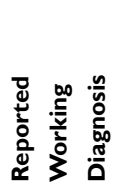 & 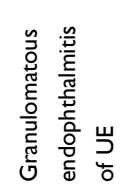 & 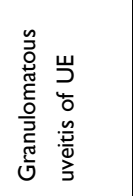 & 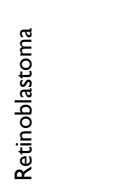 & 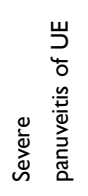 & 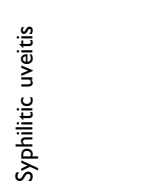 & 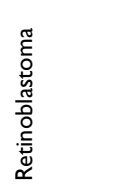 & 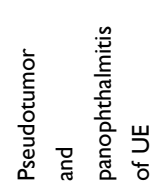 & 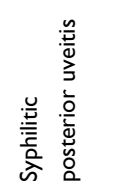 & 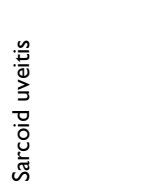 & $\widetilde{\Sigma}$ \\
\hline 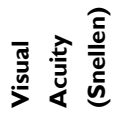 & 吕 & $\begin{array}{l}\text { ¿্ড } \\
\text { ড̀ }\end{array}$ & $\tilde{z}$ & $\tilde{z}$ & $\stackrel{\hat{z}}{\mathrm{z}}$ & 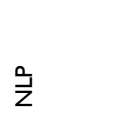 & $\tilde{\mathrm{z}}$ & $\sum_{I}$ & 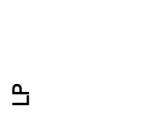 & $\tilde{\mathrm{z}}$ \\
\hline 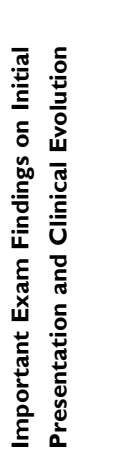 & 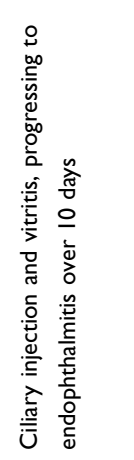 & 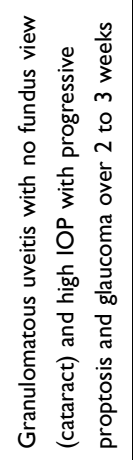 & 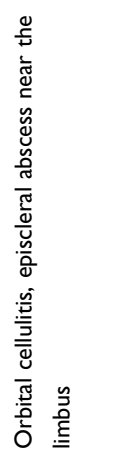 & 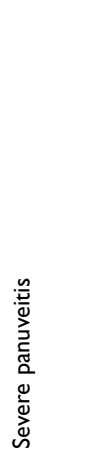 & 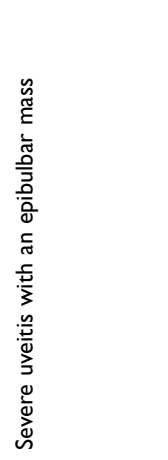 & 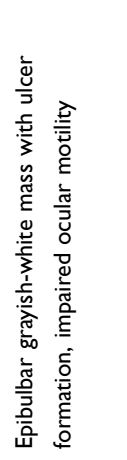 & 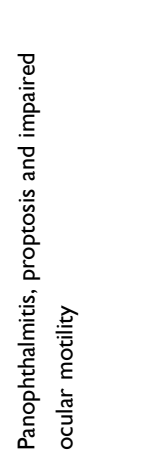 & 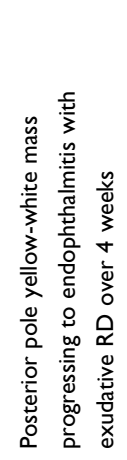 & 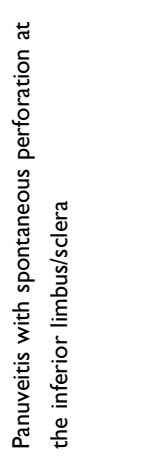 & 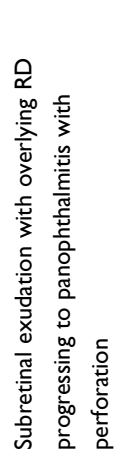 \\
\hline है & 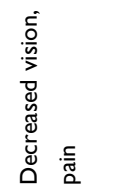 & 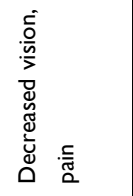 & 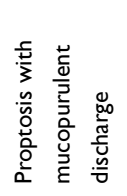 & $\tilde{z}$ & 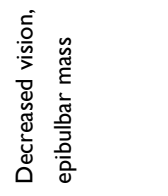 & 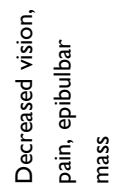 & 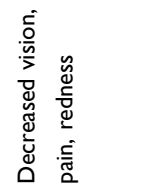 & 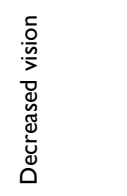 & 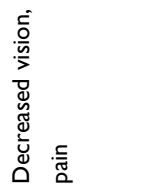 & $\widetilde{\Sigma}$ \\
\hline 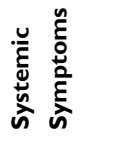 & $\stackrel{\check{\nu}}{\approx}$ & $\stackrel{\check{\nu}}{\check{\nu}}$ & ㅇ & 우 & $\stackrel{\check{\nu}}{\check{\nu}}$ & $\stackrel{\check{\varpi}}{\check{\nu}}$ & zo & $\stackrel{\check{\varpi}}{\check{\nu}}$ & $\stackrel{\circ}{z}$ & $\widetilde{n}$ \\
\hline 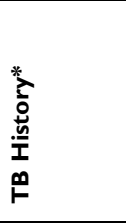 & 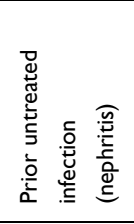 & $\stackrel{0}{z}$ & $\stackrel{0}{z}$ & $\stackrel{\circ}{z}$ & 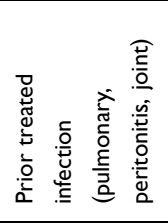 & 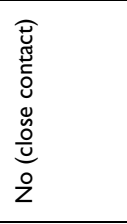 & 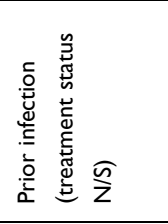 & $\stackrel{0}{z}$ & 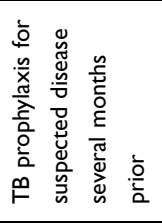 & 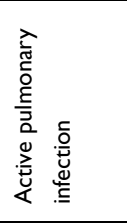 \\
\hline 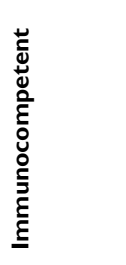 & $\stackrel{\check{\nu}}{\tau}$ & $\check{\check{\nu}}^{\breve{\Delta}}$ & $\stackrel{\check{\Perp}}{\check{\nu}}$ & $\stackrel{\mathscr{\varpi}}{\sim}$ & $\stackrel{\check{\Xi}}{\check{\nu}}$ & $\stackrel{\check{\Xi}}{\check{\nu}}$ & $\stackrel{\check{\Xi}}{\check{\nu}}$ & 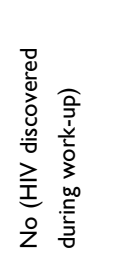 & 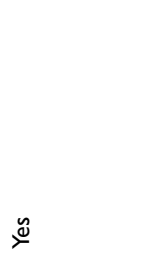 & 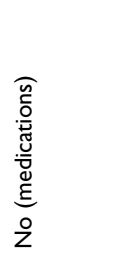 \\
\hline 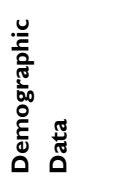 & 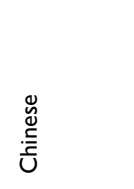 & $\widetilde{\Omega}$ & 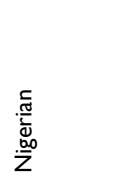 & 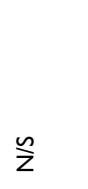 & $\widetilde{\Omega}$ & $\stackrel{n}{z}$ & $\widetilde{n}$ & 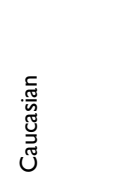 & 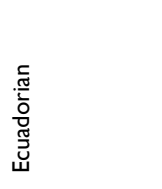 & 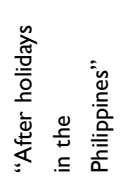 \\
\hline ڤ̊ & $\Sigma$ & $\Sigma$ & $\Sigma$ & 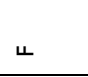 & \llcorner & $\Sigma$ & 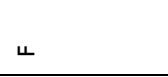 & 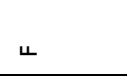 & 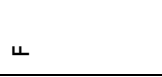 & \llcorner \\
\hline$\underset{8}{\stackrel{\circ}{8}}$ & $\hat{m}$ & $\stackrel{m}{n}$ & - & ㅇ & 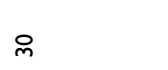 & $=$ & $\underline{n}$ & i & నे & $\stackrel{\infty}{m}$ \\
\hline$\#$ & - & $N$ & $m$ & $\sigma$ & in & ○ & $n$ & $\infty$ & $a$ & 으 \\
\hline 旁 & 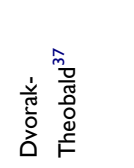 & 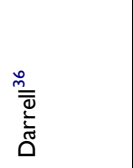 & 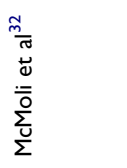 & 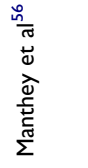 & $\begin{array}{l}\frac{\infty}{\pi} \\
\frac{\pi}{0} \\
\dot{z}\end{array}$ & & & 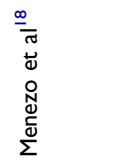 & 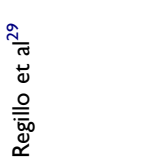 & 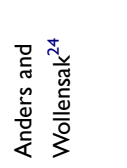 \\
\hline
\end{tabular}




\begin{tabular}{|c|c|c|c|c|c|c|c|c|c|c|c|c|}
\hline 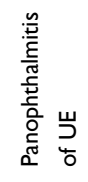 & 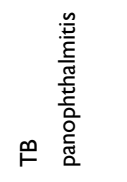 & 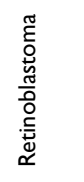 & 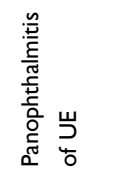 & 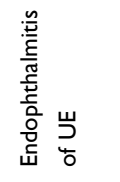 & 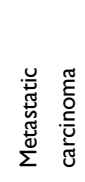 & 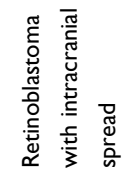 & 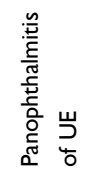 & 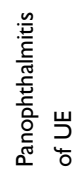 & 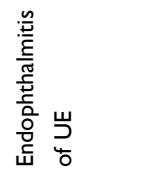 & 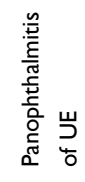 & 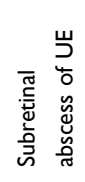 & 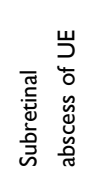 \\
\hline$\stackrel{\circ}{\circ}$ & $\stackrel{\stackrel{े}{z}}{z}$ & $\stackrel{\hat{z}}{z}$ & $\stackrel{a}{z}$ & 。্ & 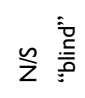 & 言 & $\stackrel{a}{z}$ & $\stackrel{9}{z}$ & a & $\widetilde{\Sigma}$ & $\tilde{z}$ & $\widetilde{\Sigma}$ \\
\hline 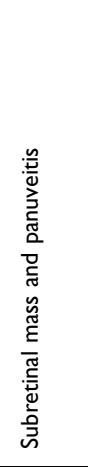 & 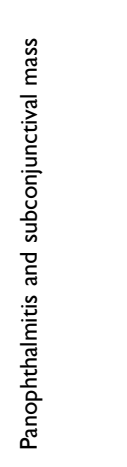 & 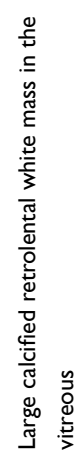 & 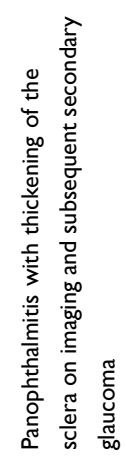 & 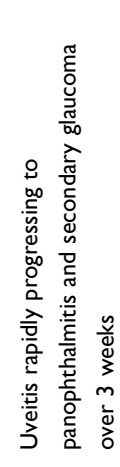 & 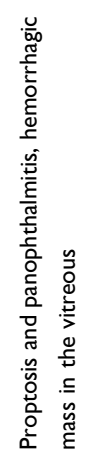 & 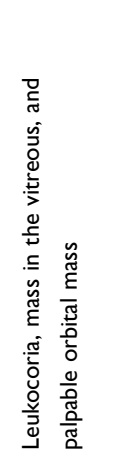 & 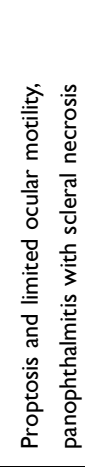 & 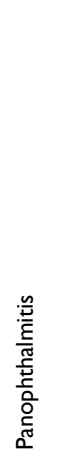 & 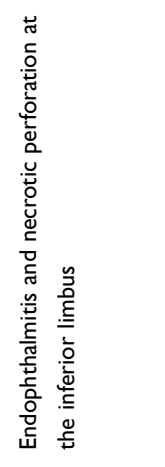 & 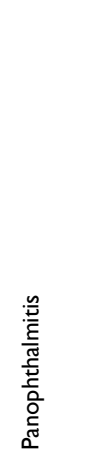 & 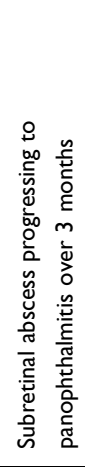 & 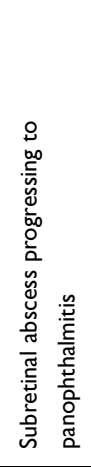 \\
\hline 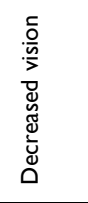 & 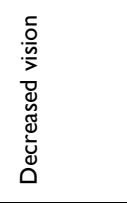 & 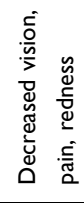 & 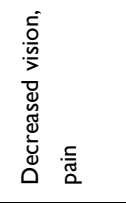 & 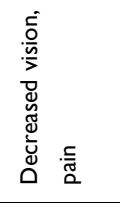 & 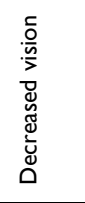 & 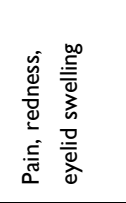 & 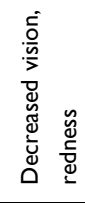 & 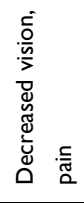 & 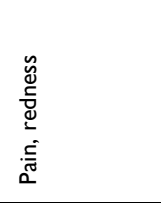 & $\tilde{z}$ & $\widetilde{\Sigma}$ & $\widetilde{\Sigma}$ \\
\hline z & $\stackrel{\mathscr{\Perp}}{\check{\nu}}$ & $\stackrel{0}{z}$ & ㅇ & $\stackrel{\tilde{y}}{x}$ & $\stackrel{\mathscr{0}}{\sigma}$ & $\stackrel{\tilde{v}}{\tau}$ & $\stackrel{\check{\varpi}}{\check{\nu}}$ & o & zo & $\tilde{z}$ & $\widetilde{\Sigma}$ & $\widetilde{z}$ \\
\hline$\stackrel{\circ}{z}$ & 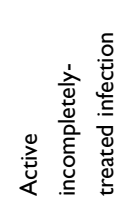 & 으 & 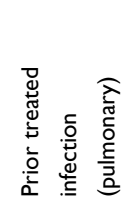 & z & 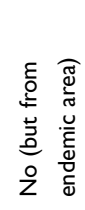 & 우 & z & $\stackrel{0}{z}$ & 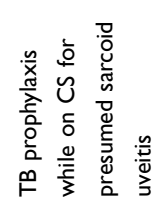 & 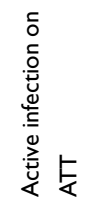 & $\stackrel{0}{z}$ & $\stackrel{\circ}{z}$ \\
\hline 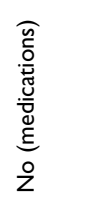 & $\stackrel{\tilde{\nu}}{\check{\nu}}$ & $\stackrel{\tilde{\nu}}{\check{\nu}}$ & $\stackrel{\tilde{\Xi}}{\tau}$ & $\stackrel{\tilde{y}}{\nu}$ & $\stackrel{\check{\varpi}}{\nu}$ & $\stackrel{\mathscr{v}}{\nu}^{\tilde{y}}$ & $\stackrel{\tilde{\Perp}}{\check{\nu}}$ & 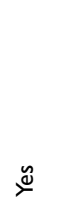 & $\stackrel{\tilde{\Xi}}{\nu}$ & $\begin{array}{l}\stackrel{S}{\text { I. }} \\
\text { Zo }\end{array}$ & $\begin{array}{l}\stackrel{\Sigma}{\mathbf{1}} \\
\text { 之o }\end{array}$ & $\begin{array}{l}\stackrel{8}{\mathbf{I}} \\
\text { 足 }\end{array}$ \\
\hline$\widetilde{\Sigma}$ & $\tilde{z}$ & $\widetilde{z}$ & $\widetilde{z}$ & $\widetilde{\Sigma}$ & 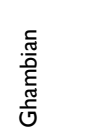 & $\widetilde{z}$ & $\widetilde{\Sigma}$ & 婿 & 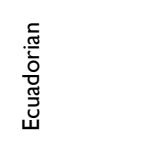 & $\tilde{z}$ & $\tilde{\mathrm{z}}$ & $\widetilde{\Sigma}$ \\
\hline ч & ч & ч & ч & $\Sigma$ & $\Sigma$ & $\Sigma$ & 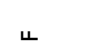 & $\Sigma$ & ч & $\Sigma$ & $\Sigma$ & $\Sigma$ \\
\hline $\mathcal{F}$ & 요 & $\infty$ & $\stackrel{n}{n}$ & @ & 요 & $\checkmark$ & $\simeq$ & $\underset{\sim}{\infty}$ & సి & ๒ & $\stackrel{m}{m}$ & $\stackrel{m}{m}$ \\
\hline$=$ & $\simeq$ & $\underline{m}$ & \pm & $\underline{n}$ & $\underline{\circ}$ & $=$ & $\underline{\infty}$ & $\underline{a}$ & i & $\bar{N}$ & A & $\approx$ \\
\hline 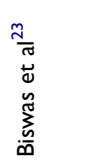 & & 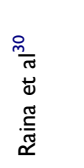 & 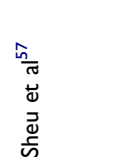 & & 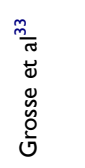 & $\begin{array}{l}\overline{\bar{m}} \overline{\bar{J}} \\
\bar{\Xi} \\
\overline{\tilde{D}}\end{array}$ & 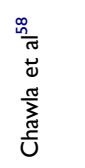 & \multicolumn{2}{|l|}{ 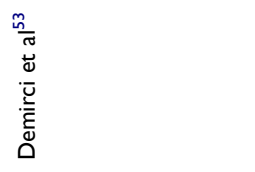 } & \multicolumn{3}{|l|}{ 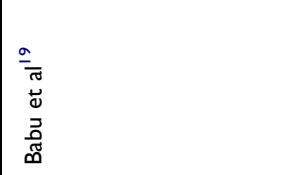 } \\
\hline
\end{tabular}




\begin{tabular}{|c|c|c|c|c|c|c|c|c|c|c|c|c|c|}
\hline 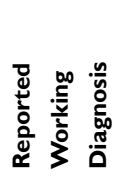 & 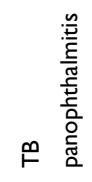 & $\widetilde{\Sigma}$ & $\widetilde{\Sigma}$ & $\tilde{z}$ & $\widetilde{\Sigma}$ & $\widetilde{\sim}$ & $\widetilde{n}$ & $\widetilde{n}$ & $\widetilde{\Sigma}$ & $\widetilde{\tilde{Z}}$ & 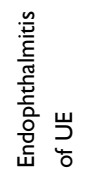 & 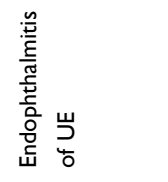 & 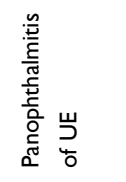 \\
\hline 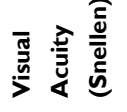 & $\stackrel{\hat{z}}{\mathrm{z}}$ & $\widetilde{\Sigma}$ & $\widetilde{\Sigma}$ & $\widetilde{n}$ & $\widetilde{\Sigma}$ & $\stackrel{\sim}{z}$ & $\widetilde{\Sigma}$ & $\widetilde{\Sigma}$ & $\widetilde{\Sigma}$ & a & a & $\stackrel{\hat{z}}{\mathrm{z}}$ & $\stackrel{\hat{z}}{\mathrm{z}}$ \\
\hline 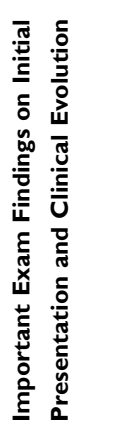 & 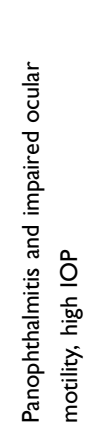 & 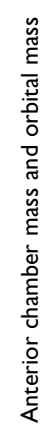 & 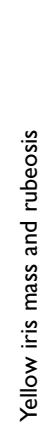 & 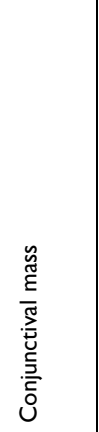 & 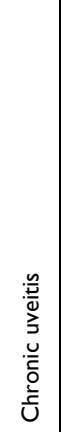 & \begin{tabular}{l|}
$\frac{c}{0}$ \\
$\frac{0}{2}$ \\
$\overline{0}$ \\
$\tilde{0}$ \\
0
\end{tabular} & 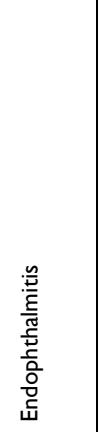 & 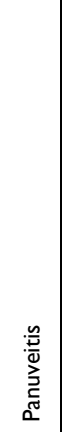 & 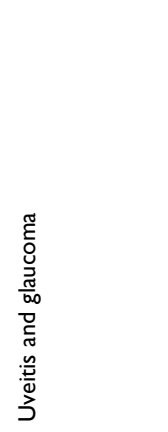 & 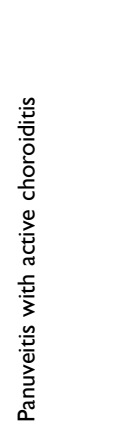 & 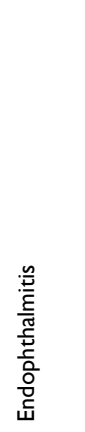 & 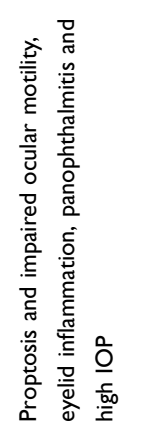 & 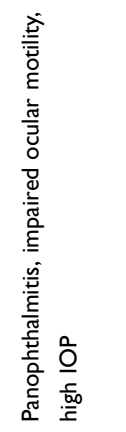 \\
\hline 尊 & 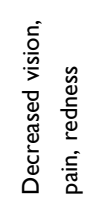 & $\widetilde{\Sigma}$ & $\widetilde{\Sigma}$ & $\widetilde{\Sigma}$ & $\widetilde{\Sigma}$ & $\stackrel{\sim}{z}$ & $\widetilde{\Sigma}$ & $\widetilde{\Sigma}$ & $\widetilde{\Sigma}$ & 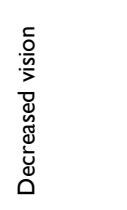 & 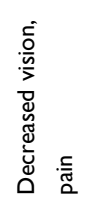 & 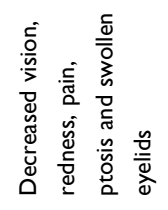 & 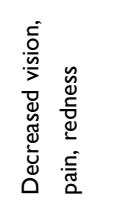 \\
\hline 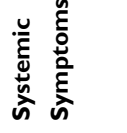 & $\stackrel{0}{z}$ & $\widetilde{\Sigma}$ & $\tilde{z}$ & $\widetilde{\Sigma}$ & $\widetilde{\Sigma}$ & $\stackrel{\sim}{z}$ & $\widetilde{n}$ & $\widetilde{\mathrm{z}}$ & $\widetilde{z}$ & 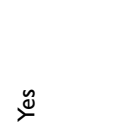 & zo & $\stackrel{\check{g}}{\check{E}}$ & $\stackrel{\check{\varpi}}{\tau}$ \\
\hline 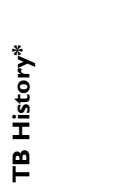 & zo & $\widetilde{z}$ & $\widetilde{\Sigma}$ & $\widetilde{\Sigma}$ & $\widetilde{\Sigma}$ & $\stackrel{\sim}{z}$ & $\widetilde{z}$ & $\widetilde{\Sigma}$ & 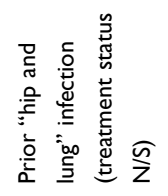 & 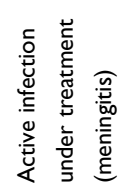 & zo & $\stackrel{0}{z}$ & 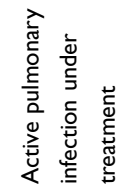 \\
\hline 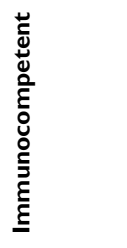 & $\stackrel{\tilde{s}}{\tau}$ & $\widetilde{\Sigma}$ & $\widetilde{z}$ & $\widetilde{\Sigma}$ & $\widetilde{\Sigma}$ & $\stackrel{n}{z}$ & $\widetilde{\Sigma}$ & $\widetilde{\Sigma}$ & $\widetilde{\Sigma}$ & $\stackrel{\check{\nu}}{\nu}$ & $\stackrel{\check{\nu}}{\nsim}$ & 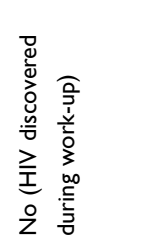 & $\stackrel{\check{\nu}}{\sim}$ \\
\hline 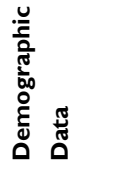 & 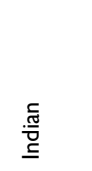 & 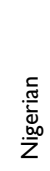 & 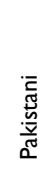 & 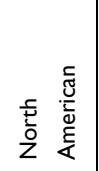 & 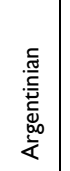 & 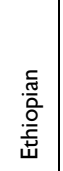 & 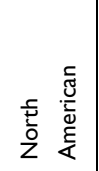 & 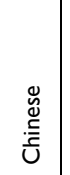 & $\begin{array}{l}\text { 旁 } \\
\text { 总 }\end{array}$ & $\widetilde{z}$ & 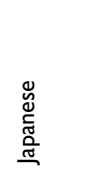 & $\overline{\bar{\sigma}}$ & $\widetilde{z}$ \\
\hline ڤั & $\Sigma$ & 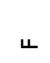 & ч & น & $\Sigma$ & $\Sigma$ & $\Sigma$ & $\Sigma$ & $\Sigma$ & $\Sigma$ & $\Sigma$ & $\Sigma$ & $\Sigma$ \\
\hline$\stackrel{8}{\&}$ & \pm & $m$ & $\underline{\infty}$ & $\leq$ & $\infty$ & $\underline{\underline{n}}$ & ถุ & बి & $\bar{\sigma}$ & $\grave{\lambda}$ & $\bar{\infty}$ & $\stackrel{\infty}{m}$ & $\stackrel{\sim}{2}$ \\
\hline \# & $\dot{A}$ & $\stackrel{\sim}{\sim}$ & $\stackrel{\sim}{2}$ & $\hat{\sim}$ & $\stackrel{\infty}{\sim}$ & ते & ০ & $\bar{m}$ & $\tilde{m}$ & $\stackrel{m}{m}$ & $\stackrel{+}{m}$ & $\stackrel{\sim}{m}$ & $\stackrel{m}{\circ}$ \\
\hline $\begin{array}{l}\frac{1}{2} \\
\frac{\dot{z}}{\alpha}\end{array}$ & 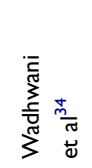 & 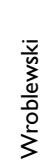 & & & & & & & & 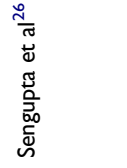 & 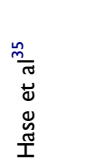 & 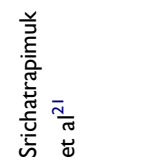 & 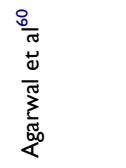 \\
\hline
\end{tabular}




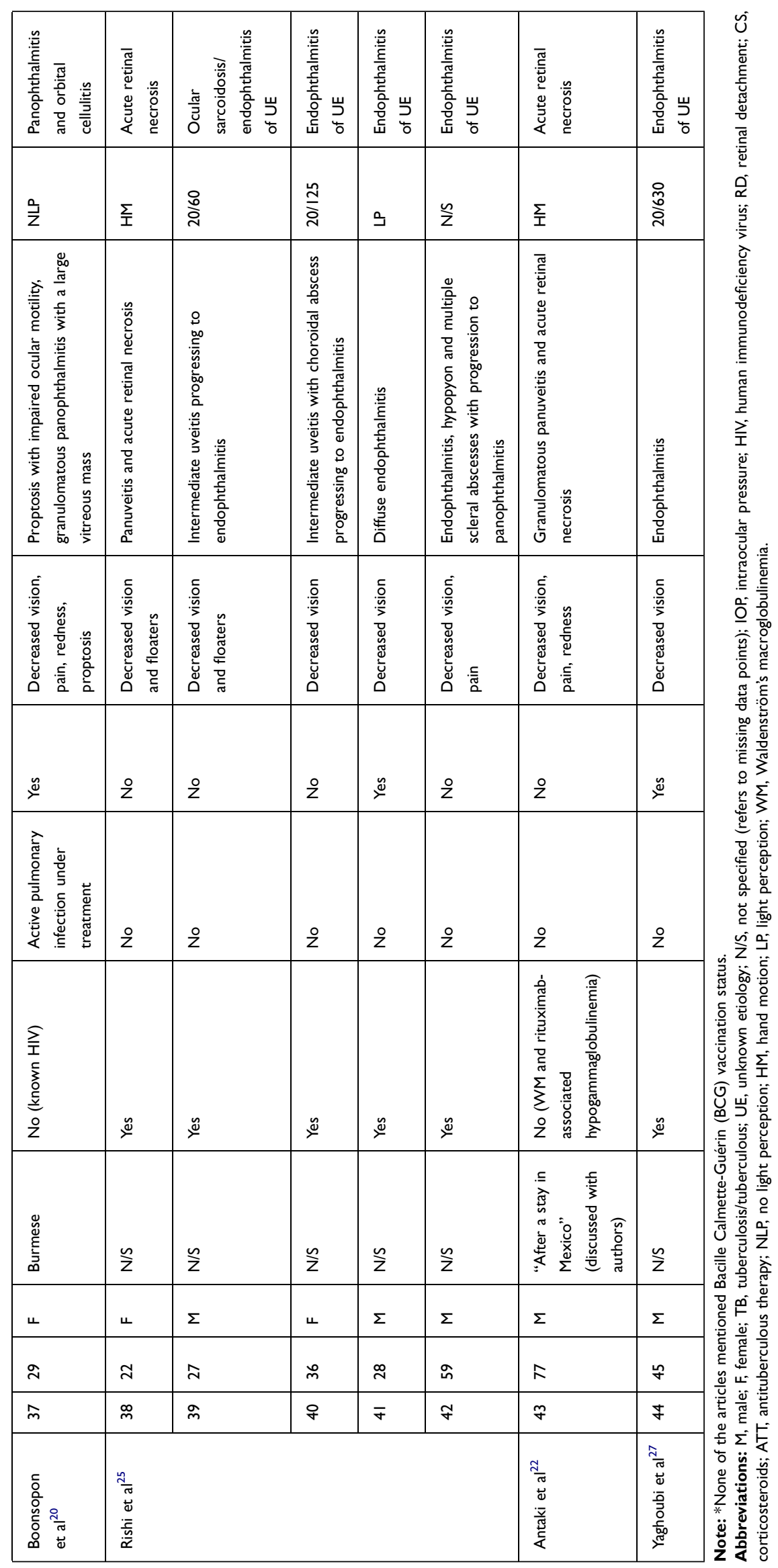




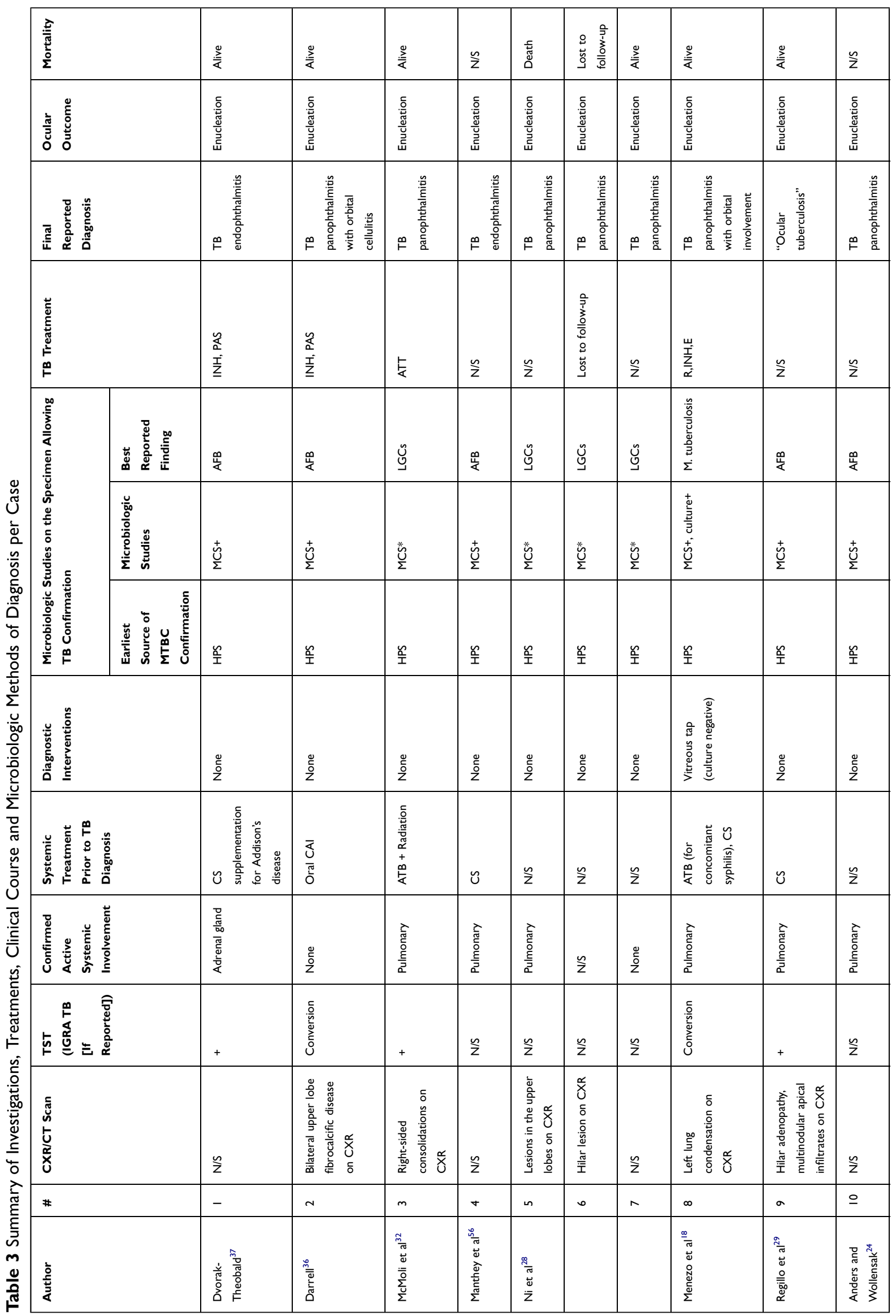




\begin{tabular}{|c|c|c|c|c|c|c|c|c|c|c|}
\hline$\stackrel{\stackrel{2}{*}}{4}$ & $\stackrel{\frac{2}{\alpha}}{\frac{2}{\alpha}}$ & $\stackrel{\frac{\partial}{4}}{\frac{2}{4}}$ & $\frac{\stackrel{2}{4}}{4}$ & $\stackrel{\frac{\partial}{4}}{\frac{2}{\alpha}}$ & $\stackrel{\frac{0}{4}}{\frac{3}{4}}$ & $\stackrel{\frac{2}{4}}{\stackrel{2}{\alpha}}$ & $\frac{⿱ 乛}{\frac{2}{\alpha}}$ & $\stackrel{\frac{2}{4}}{\frac{\pi}{4}}$ & $\stackrel{\frac{2}{4}}{\frac{\pi}{4}}$ & $\stackrel{\frac{2}{4}}{\frac{3}{4}}$ \\
\hline 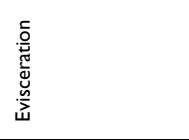 & 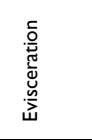 & 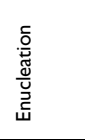 & 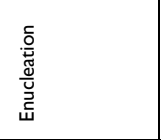 & 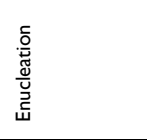 & 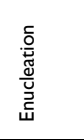 & 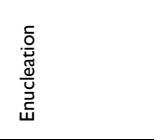 & 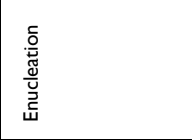 & 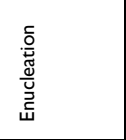 & 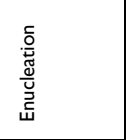 & 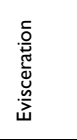 \\
\hline 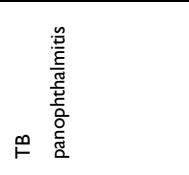 & 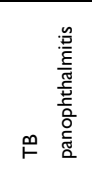 & 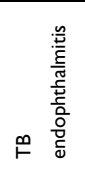 & 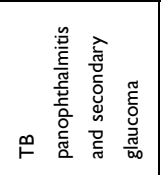 & 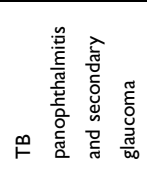 & 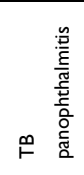 & 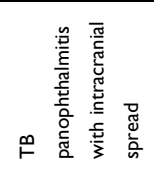 & 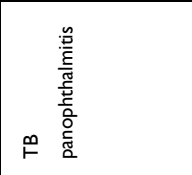 & 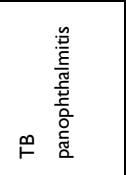 & 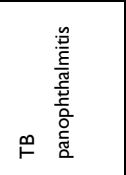 & 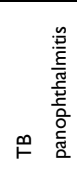 \\
\hline 卡 & 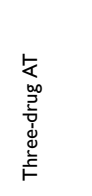 & 安 & $\frac{\underline{\underline{\mu}}}{\underline{\alpha}}$ & 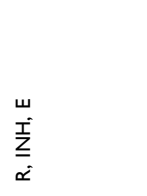 & $\frac{\underline{\omega}}{\underline{\alpha}}$ & 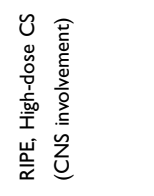 & 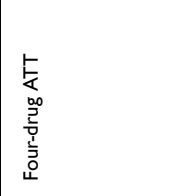 & 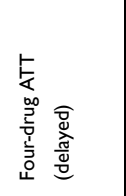 & $\begin{array}{l}\widehat{\underline{\lambda}} \\
\underline{\underline{O}} \\
\underline{\underline{I}}\end{array}$ & 卡 \\
\hline 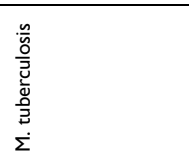 & 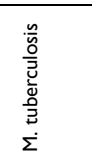 & 兑 & 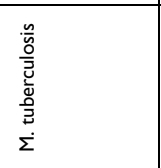 & 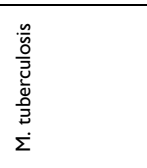 & 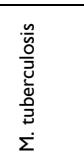 & 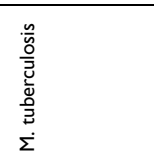 & U్ & 离 & 兑 & 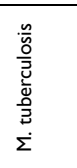 \\
\hline 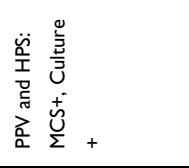 & 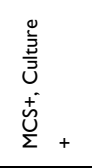 & $\stackrel{+}{\stackrel{U}{\Sigma}}$ & 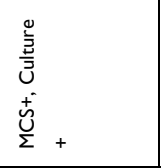 & 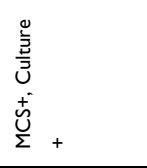 & 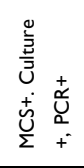 & 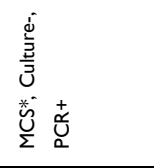 & $\begin{array}{l}\text { 苛 } \\
\end{array}$ & $\begin{array}{l}+ \\
\Sigma \\
\Sigma\end{array}$ & 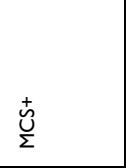 & $\begin{array}{l}+ \\
+ \\
0 \\
0 \\
+ \\
\dot{U} \\
\Sigma\end{array}$ \\
\hline 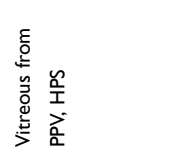 & 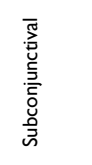 & 苗 & 蒫 & 茥 & 品 & $\stackrel{\breve{I}}{\underline{I}}$ & 茥 & 茥 & $\stackrel{\check{0}}{\underline{I}}$ & $\stackrel{\dddot{a}}{\underline{I}}$ \\
\hline 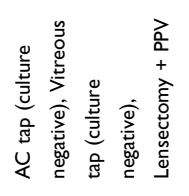 & 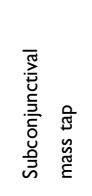 & $\begin{array}{l}0 \\
\stackrel{5}{5} \\
z\end{array}$ & 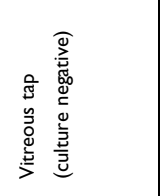 & 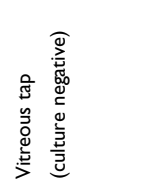 & $\stackrel{\circ}{\frac{0}{5}}$ & 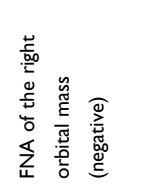 & 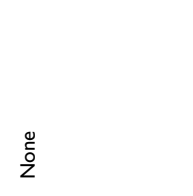 & 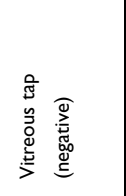 & 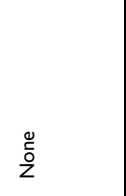 & $\tilde{z}$ \\
\hline $\begin{array}{l}0 \\
\frac{1}{4} \\
+ \\
y\end{array}$ & y & 臬 & $\tilde{z}$ & 䎹 & $\widetilde{z}$ & 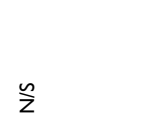 & 妥 & $y$ & $y$ & 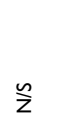 \\
\hline $\begin{array}{l}\frac{0}{5} \\
z \\
z\end{array}$ & 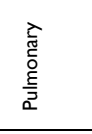 & $\begin{array}{l}\frac{0}{5} \\
z \\
\end{array}$ & 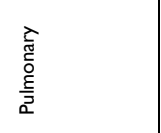 & 离竞 & $\frac{\text { 离 }}{\underline{\underline{\underline{\underline{\nu}}}}}$ & $\sum_{u}^{n}$ & 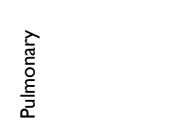 & 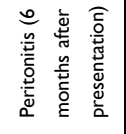 & $\stackrel{\circ}{\frac{0}{5}}$ & 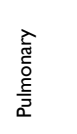 \\
\hline+ & + & + & $\widehat{\Sigma}$ & $\tilde{\Sigma}$ & + & + & . & . & + & 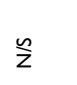 \\
\hline$\stackrel{n}{z}$ & 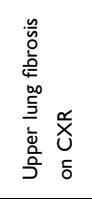 & 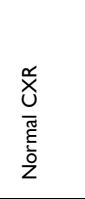 & 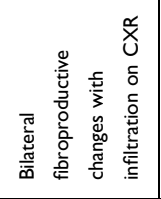 & 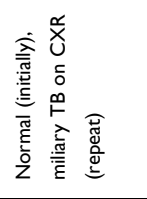 & 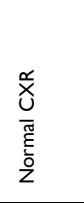 & 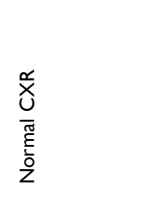 & 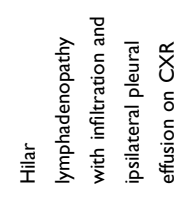 & $\begin{array}{l}\tilde{X} \\
\frac{\tilde{X}}{\tilde{E}} \\
\underline{\tilde{g}} \\
z\end{array}$ & 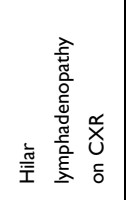 & $\tilde{z}$ \\
\hline$=$ & $\simeq$ & $\underline{m}$ & \pm & $\underline{\sim}$ & $\underline{0}$ & $\simeq$ & $\underline{\infty}$ & 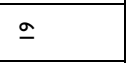 & i & $\bar{\lambda}$ \\
\hline 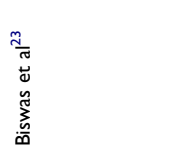 & & 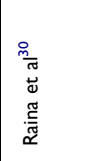 & 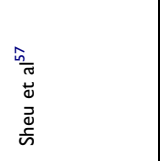 & & 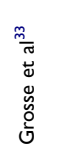 & 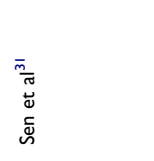 & 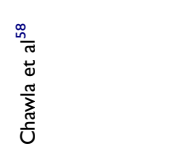 & 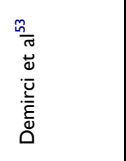 & & $\begin{array}{l}\frac{\sigma}{\bar{\sigma}} \\
\bar{\Delta} \\
\bar{z} \\
\overline{0}\end{array}$ \\
\hline
\end{tabular}




\begin{tabular}{|c|c|c|c|c|c|c|c|c|c|c|c|c|c|}
\hline \multicolumn{2}{|l|}{ 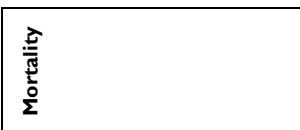 } & $\stackrel{\frac{⿱ 亠 凶}{\alpha}}{4}$ & $\stackrel{\frac{0}{4}}{\frac{1}{4}}$ & $\stackrel{\frac{0}{\alpha}}{\frac{3}{\alpha}}$ & $\tilde{\tilde{z}}$ & $\widetilde{\Omega}_{\mathbf{Z}}$ & $\tilde{\Sigma}$ & $\tilde{z}$ & $\tilde{z}$ & $\tilde{\underline{z}}$ & $\tilde{\underline{z}}$ & $\widetilde{\tilde{z}}$ & 善 \\
\hline \multicolumn{2}{|l|}{ 竞 } & 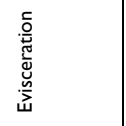 & 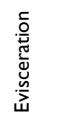 & 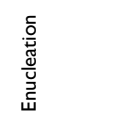 & 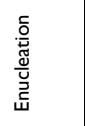 & 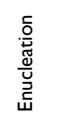 & 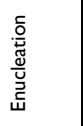 & 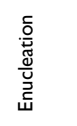 & 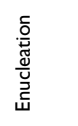 & 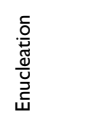 & 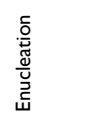 & 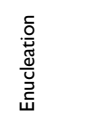 & Ü \\
\hline \multicolumn{2}{|c|}{ 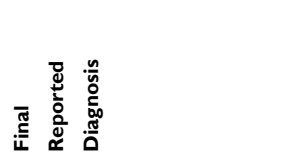 } & 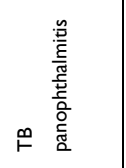 & 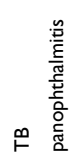 & 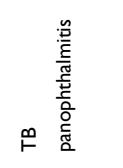 & 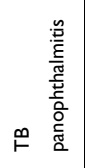 & 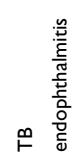 & 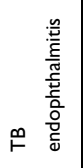 & 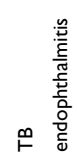 & 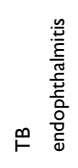 & 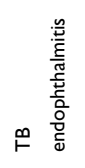 & م & 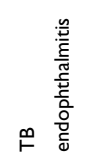 & 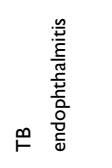 \\
\hline \multicolumn{2}{|l|}{ 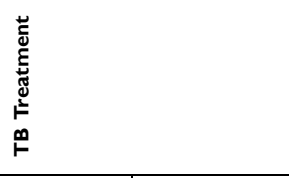 } & 点 & 安 & 奌 & $\tilde{\mathrm{z}}$ & $\widetilde{\tilde{z}}$ & $\widetilde{\Sigma}$ & $\tilde{\mathrm{Z}}$ & $\tilde{z}$ & $\tilde{z}$ & $\tilde{z}$ & $\tilde{z}$ & 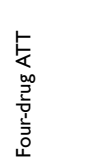 \\
\hline \multirow{3}{*}{ 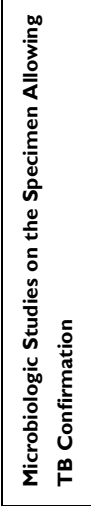 } & 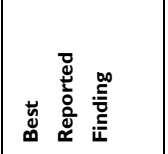 & פ్ & 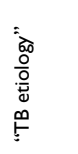 & 兑 & 政 & 这 & 这 & 兑 & 正 & 昰 & 这 & 这 & 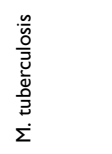 \\
\hline & 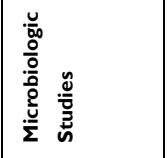 & 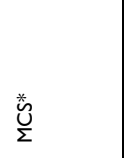 & $\tilde{\Sigma}$ & $\begin{array}{l}+ \\
\stackrel{U}{U}\end{array}$ & 憂 & $\begin{array}{l}+ \\
\stackrel{U}{U}\end{array}$ & 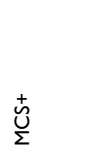 & $\begin{array}{l}\dot{U} \\
\Sigma\end{array}$ & 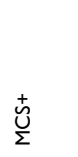 & 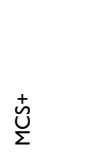 & 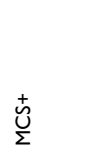 & $\begin{array}{l}+ \\
\stackrel{U}{U}\end{array}$ & $\begin{array}{l}+ \\
\stackrel{ \pm}{0} \\
\square\end{array}$ \\
\hline & 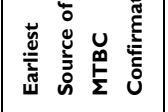 & 茥 & 高 & 茴 & 㟔 & 琹 & 䒘 & 垔 & 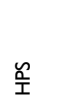 & 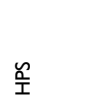 & $\frac{\tilde{a}}{\underline{1}}$ & 苗 & $\begin{array}{l}\text { a } \\
\text { U⿺ }\end{array}$ \\
\hline \multicolumn{2}{|l|}{ 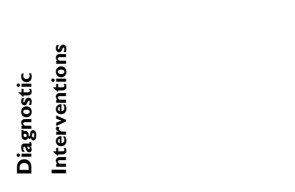 } & 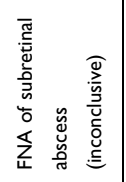 & $\frac{n}{z}$ & $\frac{\stackrel{0}{5}}{2}$ & $\tilde{\tilde{z}}$ & $\tilde{\mathrm{z}}$ & $\tilde{\mathrm{z}}$ & $\tilde{\mathrm{z}}$ & $\widetilde{\tilde{z}}$ & $\widetilde{\tilde{z}}$ & $\tilde{\mathrm{z}}$ & $\tilde{\mathrm{z}}$ & 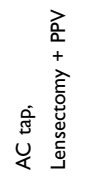 \\
\hline \multicolumn{2}{|c|}{ 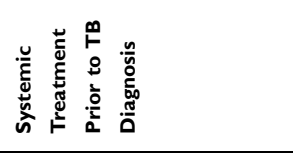 } & $\stackrel{\sim}{z}$ & $\tilde{\mathrm{z}}$ & $\tilde{\mathrm{z}}$ & $\stackrel{n}{z}$ & $\tilde{\mathrm{z}}$ & $\widetilde{\Omega}$ & $\tilde{\mathrm{z}}$ & $\tilde{\mathrm{z}}$ & $\tilde{\mathrm{z}}$ & $\tilde{z}$ & $\tilde{z}$ & $\tilde{z}$ \\
\hline \multicolumn{2}{|c|}{ 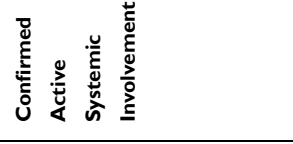 } & 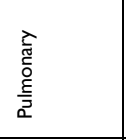 & 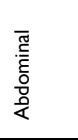 & 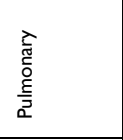 & $\stackrel{n}{z}$ & $\stackrel{n}{z}$ & $\stackrel{n}{z}$ & $\stackrel{n}{z}$ & $\frac{\Omega}{z}$ & $\stackrel{\Omega}{z}$ & $\stackrel{\sim}{z}$ & $\stackrel{n}{z}$ & 産 \\
\hline \multicolumn{2}{|c|}{ 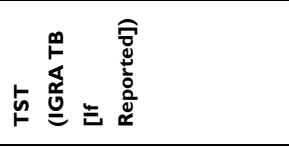 } & $\tilde{\Sigma}^{n}$ & $\stackrel{n}{z}$ & $\widetilde{\Omega}$ & $\stackrel{n}{z}$ & $\widetilde{n}$ & + & $\widetilde{\sim}$ & $\stackrel{n}{z}$ & $\widetilde{\sim}$ & $\stackrel{\sim}{z}$ & $\widetilde{\Omega}$ & $\stackrel{n}{z}$ \\
\hline \multicolumn{2}{|l|}{ 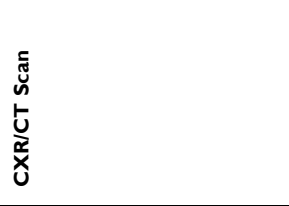 } & $\widetilde{\Omega}$ & $\stackrel{n}{z}$ & 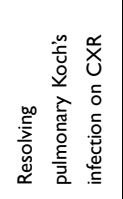 & $\tilde{\tilde{z}}$ & $\tilde{\mathrm{z}}$ & $\tilde{z}$ & $\tilde{z}$ & $\tilde{\mathrm{z}}$ & $\tilde{\mathrm{z}}$ & $\tilde{\mathrm{z}}$ & $\tilde{z}$ & 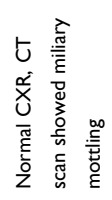 \\
\hline \multicolumn{2}{|l|}{$\#$} & $\approx$ & ๙ & $\stackrel{ \pm}{ \pm}$ & $\stackrel{2}{2}$ & $\stackrel{2}{2}$ & $\lambda$ & $\stackrel{\infty}{\infty}$ & సి & i & $\bar{m}$ & $\widetilde{m}$ & m \\
\hline \multicolumn{2}{|l|}{ 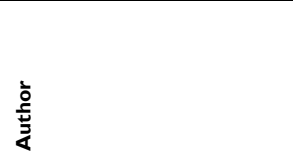 } & & & 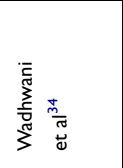 & 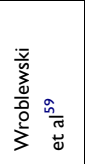 & & & & & & & & 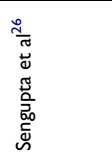 \\
\hline
\end{tabular}




\begin{tabular}{|c|c|c|c|c|c|c|c|c|c|c|c|}
\hline 旁 & $\frac{0}{\frac{3}{4}}$ & 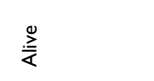 & $\frac{0}{\frac{3}{4}}$ & 旁 & 旁 & 旁 & 遂 & $\stackrel{0}{\frac{\tilde{z}}{\alpha}}$ & $\frac{0}{\frac{3}{4}}$ & $\stackrel{\frac{0}{3}}{\frac{3}{\alpha}}$ & 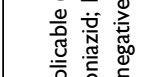 \\
\hline ఫั ڤે & 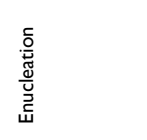 & 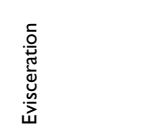 & 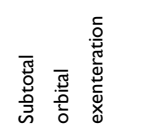 & 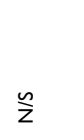 & 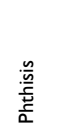 & $\frac{a}{z}$ & 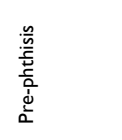 & 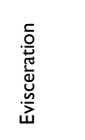 & 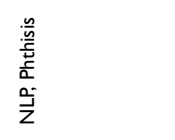 & $\tilde{\tilde{a}}$ & 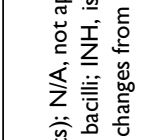 \\
\hline 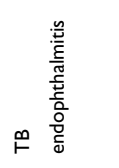 & 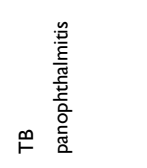 & 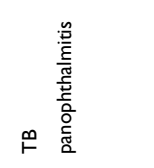 & 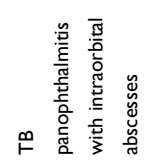 & 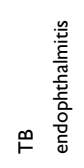 & 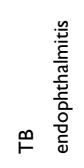 & 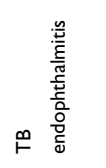 & م & 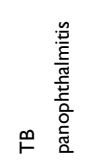 & 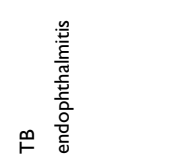 & 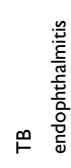 & 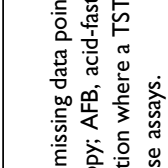 \\
\hline $\begin{array}{l}\underset{\underline{z}}{\underline{\underline{z}}} \\
\underset{\alpha}{\alpha}\end{array}$ & 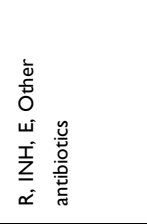 & 奌 & 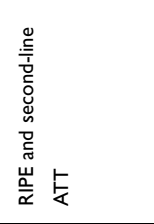 & $\frac{\underline{\underline{\underline{\alpha}}}}{\bar{\alpha}}$ & 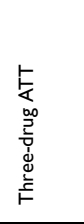 & 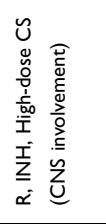 & 卡 & 安 & 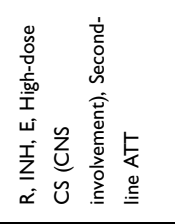 & 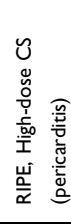 & 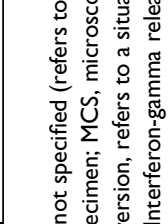 \\
\hline 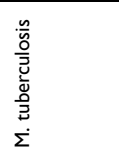 & 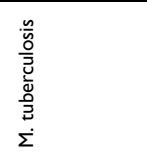 & 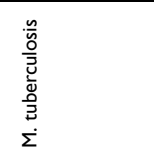 & 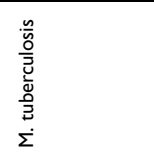 & 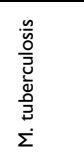 & 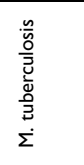 & 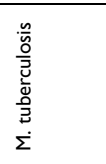 & 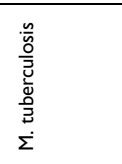 & 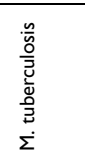 & $\begin{array}{l}\frac{n}{0} \\
\vdots \\
\Sigma \\
\Sigma\end{array}$ & 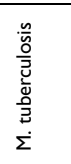 & 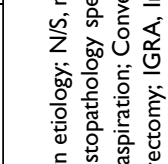 \\
\hline 产 & 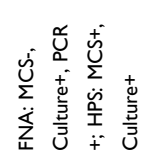 & 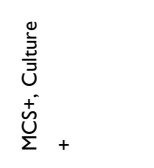 & 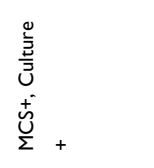 & 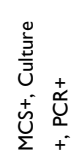 & 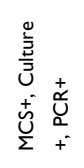 & 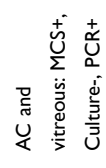 & 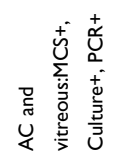 & 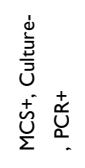 & 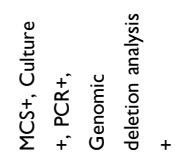 & 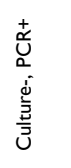 & 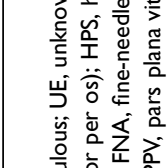 \\
\hline 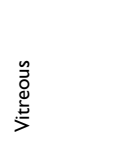 & 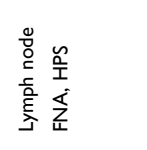 & 䓂 & 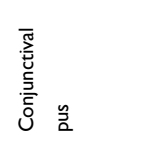 & 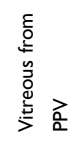 & 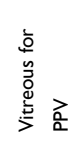 & 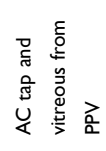 & 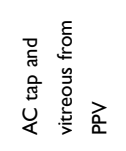 & 豆 & 品 & 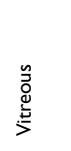 & 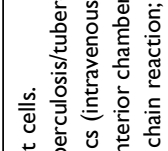 \\
\hline aㅗㅁ & 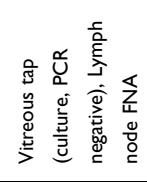 & $\begin{array}{l}\text { o } \\
\text { s. }\end{array}$ & 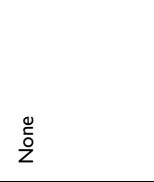 & aㅗㅁ & 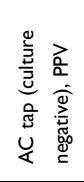 & 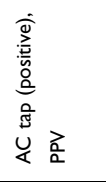 & 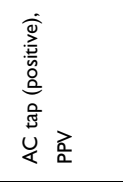 & 造 & a & 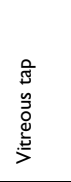 & 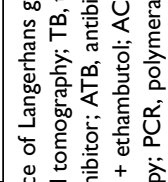 \\
\hline$y$ & 晏 & $\widetilde{\sim}$ & 曼 & 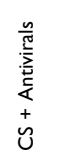 & 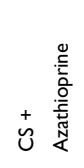 & $\stackrel{\varrho}{z}$ & y & $\begin{array}{l}0 \\
\frac{9}{5} \\
+ \\
\text { y }\end{array}$ & 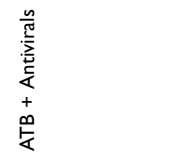 & $\stackrel{n}{z}$ & 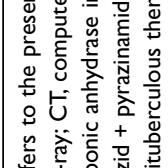 \\
\hline 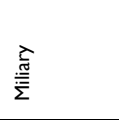 & そ & 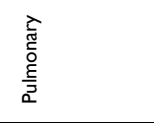 & 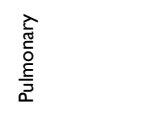 & $\frac{0}{5}$ & $\begin{array}{l}\frac{0}{5} \\
z\end{array}$ & 產 & 耪 & 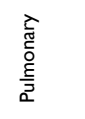 & 耪 & 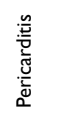 & 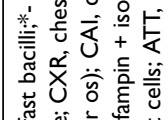 \\
\hline$\stackrel{n}{z}$ & + & $\widehat{\Sigma}$ & 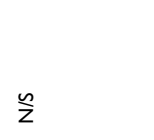 & $\begin{array}{l}\frac{\infty}{6} \\
\frac{8}{0} \\
\stackrel{0}{0}\end{array}$ & 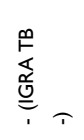 & 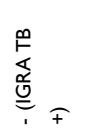 & 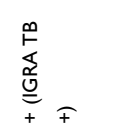 & 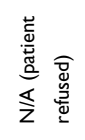 & & $\stackrel{n}{z}$ & 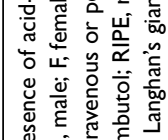 \\
\hline 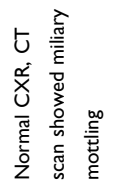 & 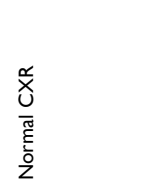 & 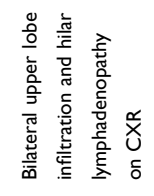 & $\tilde{n}$ & 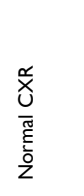 & 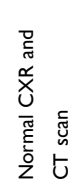 & 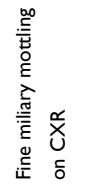 & 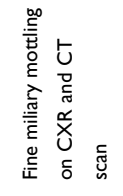 & 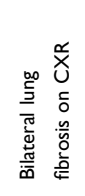 & 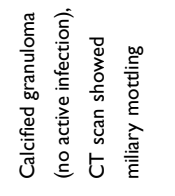 & $\begin{array}{l}\frac{0}{x} \\
\frac{x}{\tilde{m}} \\
\frac{\bar{g}}{2}\end{array}$ & 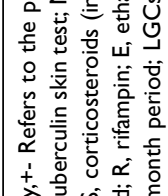 \\
\hline 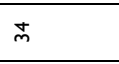 & $\stackrel{m}{m}$ & m & $\hat{m}$ & $\stackrel{\infty}{\infty}$ & o & q & $F$ & $q$ & q & F & \\
\hline 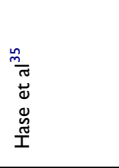 & 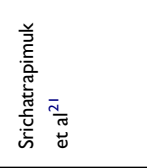 & 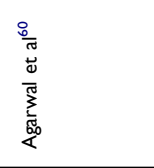 & 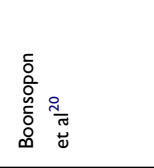 & 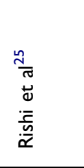 & & & & & 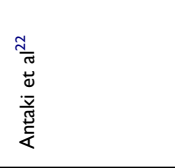 & 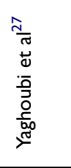 & 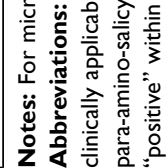 \\
\hline
\end{tabular}


Table 4 Summary of Demographic Data and Past Medical History

\begin{tabular}{|c|c|c|}
\hline Characteristics & $\begin{array}{l}\text { Number of } \\
\text { Patients/ } \\
\text { Value }\end{array}$ & Percentage \\
\hline \multicolumn{3}{|l|}{ Age } \\
\hline Adult & $33 / 44$ & $75.0 \%$ \\
\hline Pediatric & $\mathrm{II} / 44$ & $25.0 \%$ \\
\hline Mean age (SD) & $33.8(21.0)$ & \\
\hline Median age (IQR) & $29.5(27)$ & \\
\hline Range & $\begin{array}{l}\mathrm{I}-8 \mathrm{I} \text { years } \\
\text { old }\end{array}$ & \\
\hline \multicolumn{3}{|l|}{ Sex } \\
\hline Male & $26 / 44$ & $59.1 \%$ \\
\hline Female & $18 / 44$ & $40.9 \%$ \\
\hline \multicolumn{3}{|l|}{ Immune status } \\
\hline Immunocompetent & $27 / 36$ & $75.0 \%$ \\
\hline Immunocompromised & $9 / 36$ & $25.0 \%$ \\
\hline Secondary to medical diagnosis & $7 / 9$ & $77.8 \%$ \\
\hline $\begin{array}{l}\text { Secondary to immunosuppressive } \\
\text { therapy }\end{array}$ & $2 / 9$ & $22.2 \%$ \\
\hline Not specified & $8 / 44$ & \\
\hline \multicolumn{3}{|l|}{$\begin{array}{l}\text { Previous diagnosis of } \\
\text { tuberculosis }\end{array}$} \\
\hline None & $24 / 38$ & $63.2 \%$ \\
\hline Active infection & $6 / 38$ & $15.8 \%$ \\
\hline Prior/remote infection & $6 / 38$ & $15.8 \%$ \\
\hline On TB prophylaxis & $2 / 38$ & $5.3 \%$ \\
\hline Not specified & $6 / 44$ & \\
\hline
\end{tabular}

Abbreviations: TB, tuberculosis/tuberculous; SD, standard deviation; IQR, interquartile range.

One or multiple diagnostic interventions (total of 24) were obtained in 17/34 patients (50.0\%). Among those, $13 / 24(54.2 \%)$ interventions yielded a positive result. In the remaining 17 cases, the authors reported no diagnostic interventions before removal of the eye. A vitreous tap was obtained in $7 / 34$ cases $(20.6 \%)$ and an anterior chamber (AC) tap was performed in 5/34 cases (14.7\%). Pars plana vitrectomy (PPV) was carried out in $8 / 34$ cases $(23.5 \%)$. Regarding diagnostic yield, comparing the different interventions, the rate of positivity was $1 / 7$ (14.3\%) for vitreous tap, $3 / 5(60.0 \%)$ for AC tap and 7/8 (87.5\%) for PPV. Case-by-case descriptions are detailed in Table 3.

Table 7 describes the diagnostic yield of culture versus PCR in cases where both tests were performed. PCRs were positive in 15/16 cases (93.75\%) and mycobacterial cultures were positive in $9 / 16$ cases $(56.25 \%)$. Considering PCR as a reference, the sensitivity and specificity of
Table 5 Summary of Clinical Characteristics and Working Diagnoses

\begin{tabular}{|c|c|c|}
\hline Characteristics & $\begin{array}{l}\text { Number of } \\
\text { Patients }\end{array}$ & Percentage \\
\hline \multicolumn{3}{|l|}{$\begin{array}{l}\text { Principal presenting } \\
\text { symptoms* }\end{array}$} \\
\hline Decreased vision & $28 / 31$ & $90.3 \%$ \\
\hline Ocular pain and/or headache & $|8 / 3|$ & $58.1 \%$ \\
\hline Redness & $10 / 31$ & $32.3 \%$ \\
\hline Abnormal protrusion of the eye & $2 / 31$ & $6.5 \%$ \\
\hline Floaters & $2 / 31$ & $6.5 \%$ \\
\hline Epibulbar mass & $2 / 31$ & $6.5 \%$ \\
\hline Eyelid swelling & $2 / 31$ & $6.5 \%$ \\
\hline Mucopurulent discharge & $|/ 3|$ & $3.2 \%$ \\
\hline Not specified & $13 / 44$ & \\
\hline \multicolumn{3}{|l|}{$\begin{array}{l}\text { Systemic symptoms prior to } \\
\text { presentation }\end{array}$} \\
\hline Present & $16 / 32$ & $50.0 \%$ \\
\hline Absent & $16 / 32$ & $50.0 \%$ \\
\hline Not specified & $12 / 44$ & \\
\hline \multicolumn{3}{|l|}{$\begin{array}{l}\text { Duration of symptoms prior to } \\
\text { presentation }\end{array}$} \\
\hline Less than one week & $5 / 30$ & $16.7 \%$ \\
\hline Between one week and four weeks & $6 / 30$ & $20.0 \%$ \\
\hline $\begin{array}{l}\text { Between one month and three } \\
\text { months }\end{array}$ & $15 / 30$ & $50.0 \%$ \\
\hline More than three months & $4 / 30$ & $13.3 \%$ \\
\hline Not specified & $14 / 44$ & \\
\hline \multicolumn{3}{|l|}{ Visual acuity } \\
\hline Better than $20 / 200$ & $4 / 27$ & $14.8 \%$ \\
\hline $20 / 200$ and worse but better than & $3 / 27$ & $11.1 \%$ \\
\hline \multicolumn{3}{|l|}{$\mathrm{HM}$} \\
\hline $\mathrm{HM}$ & $3 / 27$ & $11.1 \%$ \\
\hline LP & $5 / 27$ & $18.5 \%$ \\
\hline NLP & $12 / 27$ & $44.4 \%$ \\
\hline Not specified & $17 / 44$ & \\
\hline \multicolumn{3}{|l|}{$\begin{array}{l}\text { Important examination } \\
\text { findings/clinical features* }\end{array}$} \\
\hline Purulent intraocular inflammation & $44 / 44$ & $100 \%$ \\
\hline Impaired ocular motility & $7 / 44$ & $15.9 \%$ \\
\hline $\begin{array}{l}\text { Extraocular mass or scleral } \\
\text { abscess formation }\end{array}$ & $7 / 44$ & $15.9 \%$ \\
\hline $\begin{array}{l}\text { High intraocular pressure or } \\
\text { glaucoma }\end{array}$ & $6 / 44$ & $13.6 \%$ \\
\hline Proptosis & $6 / 44$ & $13.6 \%$ \\
\hline Perforation & $3 / 44$ & $6.8 \%$ \\
\hline Necrotizing retinitis & $2 / 44$ & $4.5 \%$ \\
\hline $\begin{array}{l}\text { Initial working diagnosis } \\
\text { Endophthalmitis of unknown } \\
\text { etiology }\end{array}$ & $10 / 34$ & $29.4 \%$ \\
\hline
\end{tabular}

(Continued) 
Table 5 (Continued).

\begin{tabular}{|c|l|l|}
\hline Characteristics & $\begin{array}{l}\text { Number of } \\
\text { Patients }\end{array}$ & Percentage \\
\hline $\begin{array}{c}\text { Versus ocular sarcoidosis } \\
\text { Panophthalmitis of unknown } \\
\text { etiology }\end{array}$ & $\mathrm{I} / 10$ & $10.0 \%$ \\
$\quad$ With associated pseudotumor & $\mathrm{I} / 8$ & $23.5 \%$ \\
$\quad$ With orbital cellulitis & $\mathrm{I} / 8$ & $12.5 \%$ \\
Retinoblastoma and other ocular & $5 / 34$ & $12.5 \%$ \\
malignancy & & $14.7 \%$ \\
Syphilitic uveitis & $2 / 34$ & \\
Subretinal abscess of unknown & $2 / 34$ & $5.9 \%$ \\
etiology & & $5.9 \%$ \\
TB panophthalmitis & $2 / 34$ & $5.9 \%$ \\
Acute retinal necrosis & $2 / 34$ & $5.9 \%$ \\
Granulomatous uveitis of & $\mathrm{I} / 34$ & $2.9 \%$ \\
unknown etiology & & \\
Severe panuveitis of unknown & $\mathrm{I} / 34$ & $2.9 \%$ \\
etiology & & \\
Sarcoid uveitis & $\mathrm{I} / 34$ & $2.9 \%$ \\
Not specified & $10 / 44$ & \\
\hline
\end{tabular}

Note: *Categories not mutually exclusive.

Abbreviations: HM, hand motion; LP, light perception; NLP, no light perception; HM, hand motion; TB, tuberculosis/tuberculous.

culture was $60.0 \%$ and $100 \%$, respectively. There were 6 cases of positive PCR despite a negative culture and no instances of negative PCR despite a positive culture.

\section{Systemic Involvement}

Systemic involvement was reported in $28 / 35$ cases (80.0\%). Patients with an abnormal chest study (fibrosis, granuloma, calcifications and other findings) but no mention of confirmed pulmonary disease by the original authors were not considered to have lung disease. Also, we considered miliary tuberculosis only when the authors used the term "miliary". Cases with central nervous system (CNS), abdominal, peritoneal, adrenal and pericardial involvement were reported separately. Confirmed pulmonary tuberculosis occurred in 15/35 (42.8\%) of cases, miliary tuberculosis in $7 / 35$ cases $(20.0 \%)$, CNS involvement in $2 / 35$ cases $(5.7 \%)$ and abdominal and peritoneal involvement in $2 / 35$ cases $(5.7 \%)$. There was 1 case of TB adrenalitis and 1 case of TB pericarditis. ${ }^{27,37}$ All patients were started on antituberculous treatment (ATT). Treatment regimens were variable and medications included rifampin, isoniazid, pyrazinamide and
Table 6 Summary of Clinic Course, Interventions and Outcomes

\begin{tabular}{|c|c|c|}
\hline Characteristics & $\begin{array}{l}\text { Number of } \\
\text { Patients }\end{array}$ & Percentage \\
\hline \multicolumn{3}{|l|}{$\begin{array}{l}\text { Systemic treatment prior to TB } \\
\text { diagnosis }\end{array}$} \\
\hline Antibiotics alone & $5 / 21$ & $23.8 \%$ \\
\hline Antibiotics with corticosteroids & $3 / 21$ & $14.3 \%$ \\
\hline Antibiotics with antivirals & $|/ 2|$ & $4.8 \%$ \\
\hline Antibiotics and radiation therapy & $|/ 2|$ & $4.8 \%$ \\
\hline Corticosteroids alone & $7 / 21$ & $33.3 \%$ \\
\hline Corticosteroids with antivirals & $|/ 2|$ & $4.8 \%$ \\
\hline $\begin{array}{l}\text { Corticosteroids with other } \\
\text { immunosuppressants }\end{array}$ & $|/ 2|$ & $4.8 \%$ \\
\hline $\begin{array}{l}\text { Corticosteroid supplementation for } \\
\text { Addison's disease }\end{array}$ & $|/ 2|$ & $4.8 \%$ \\
\hline Oral carbonic anhydrase inhibitor & $1 / 21$ & $4.8 \%$ \\
\hline No treatment or not specified & $23 / 44$ & \\
\hline \multicolumn{3}{|l|}{ Tuberculin skin test reaction } \\
\hline Positive & $12 / 20$ & $60.0 \%$ \\
\hline Negative & $6 / 20$ & $30.0 \%$ \\
\hline Conversion & $2 / 20$ & $10.0 \%$ \\
\hline Not specified or not applicable & $24 / 44$ & \\
\hline \multicolumn{3}{|l|}{ CXR and CT } \\
\hline Abnormal & $19 / 27$ & $70.4 \%$ \\
\hline Abnormal CXR (see case-by-case details) & $13 / 19$ & $68.4 \%$ \\
\hline Normal CXR, miliary mottling on CT scan & $3 / 19$ & $15.8 \%$ \\
\hline Miliary mottling on CXR & $2 / 19$ & $10.5 \%$ \\
\hline $\begin{array}{l}\text { Normal initial CXR, abnormal } \\
\text { subsequent CXR }\end{array}$ & $1 / 19$ & $5.3 \%$ \\
\hline Normal & $8 / 27$ & $29.6 \%$ \\
\hline Normal CXR & $7 / 8$ & $87.5 \%$ \\
\hline Normal CXR and CT scan & $1 / 8$ & $12.5 \%$ \\
\hline Not specified & $17 / 44$ & \\
\hline \multicolumn{3}{|l|}{ Diagnostic interventions* } \\
\hline Vitreous tap & $7 / 34$ & $20.6 \%$ \\
\hline Culture or PCR negative & $6 / 7$ & $85.7 \%$ \\
\hline Culture or PCR positive & $1 / 7$ & $14.3 \%$ \\
\hline$A C$ tap & $5 / 34$ & $14.7 \%$ \\
\hline Culture or PCR negative & $2 / 5$ & $40.0 \%$ \\
\hline Culture or PCR positive & $3 / 5$ & $60.0 \%$ \\
\hline Pars plana vitrectomy & $8 / 34$ & $23.5 \%$ \\
\hline Culture or PCR negative & $1 / 8$ & $12.5 \%$ \\
\hline Culture or PCR positive & $7 / 8$ & $87.5 \%$ \\
\hline Fine-needle aspiration & $4 / 34$ & $11.8 \%$ \\
\hline Culture negative or inconclusive & $2 / 4$ & $50.0 \%$ \\
\hline Culture positive & $2 / 4$ & $50.0 \%$ \\
\hline None & $17 / 34$ & $50.0 \%$ \\
\hline Not specified & $10 / 44$ & \\
\hline \multicolumn{3}{|l|}{ Reported systemic involvement } \\
\hline Pulmonary & $15 / 35$ & $42.8 \%$ \\
\hline Miliary & $7 / 35$ & $20.0 \%$ \\
\hline $\begin{array}{l}\text { Central nervous system (not reported } \\
\text { as miliary) }\end{array}$ & $2 / 35$ & $5.7 \%$ \\
\hline
\end{tabular}

(Continued) 
Table 6 (Continued).

\begin{tabular}{|l|l|l|}
\hline Characteristics & $\begin{array}{l}\text { Number of } \\
\text { Patients }\end{array}$ & Percentage \\
\hline $\begin{array}{l}\text { Abdominal or peritoneal (not reported } \\
\text { as miliary) }\end{array}$ & $2 / 35$ & $5.7 \%$ \\
Pericarditis (not reported as miliary) & $1 / 35$ & $2.9 \%$ \\
Adrenalitis (not reported as miliary) & $1 / 35$ & $2.9 \%$ \\
None & $7 / 35$ & $20.0 \%$ \\
Not specified & $9 / 44$ & \\
\hline Final diagnosis & & \\
TB endophthalmitis & $18 / 44$ & $40.9 \%$ \\
TB panophthalmitis & $25 / 44$ & $56.8 \%$ \\
With secondary glaucoma & $2 / 25$ & $8.0 \%$ \\
With orbital involvement & $3 / 25$ & $12.0 \%$ \\
With intracranial spread & $1 / 25$ & $4.0 \%$ \\
"Ocular tuberculosis" & $1 / 44$ & $2.3 \%$ \\
\hline Ocular outcome & & \\
Enucleation & $28 / 43$ & $65.1 \%$ \\
Evisceration & $7 / 43$ & $16.3 \%$ \\
Exenteration & $1 / 43$ & $2.3 \%$ \\
Phthisis or pre-phthisis bulbi & $3 / 43$ & $7.0 \%$ \\
Visual acuity better than 20/200 & $1 / 43$ & $2.3 \%$ \\
Visual acuity 20/200 and worse & $3 / 43$ & $7.0 \%$ \\
Not specified & $1 / 44$ & $2.3 \%$ \\
\hline Mortality outcome & & \\
Alive or not specified & $43 / 44$ & $97.7 \%$ \\
Death & $1 / 44$ & $2.3 \%$ \\
\hline Note: & & \\
\hline
\end{tabular}

Note: *Categories not mutually exclusive.

Abbreviations: TB, tuberculosis/tuberculous; CXR, chest x-ray; $\mathrm{CT}$, computed tomography; PCR, polymerase chain reaction.

ethambutol, among others. Case-by-case description of the ATT is available in Table 3. Death was reported in one case. This particular case was reported by $\mathrm{Ni}$ et al in $1982 .^{28}$ The patient was a 30 -year-old female with remote pulmonary tuberculosis who was diagnosed with syphilitic uveitis on presentation. The eye was enucleated and pathology revealed Langerhans giant cells (LGCs) which can be found in both TB and syphilis. The TB etiology was therefore not recognized and the patient died 6 months after presentation from generalized tuberculosis. In the remaining cases, patients were either referred to as alive or no mention of death was made by authors.

\section{Final Diagnosis and Ocular Outcome}

TB endophthalmitis was diagnosed in 18/44 (40.9\%) of cases and TB panophthalmitis was seen in 25/44 (56.8\%) of cases. One case reported the final diagnosis as "ocular tuberculosis". 29 Ocular outcomes were generally poor with $36 / 43$ cases $(83.7 \%)$ requiring enucleation, evisceration or exenteration. Pre-phthisis or phthisis bulbi was reported in 3 cases (7.0\%), final visual acuity 20/200 and worse was seen in 3 cases $(7.0 \%)$ and a single case reported visual acuity better than 20/200 (2.3\%). Only two authors reported a favorable visual outcome. In the case reported by Hase et al, visual acuity improved from light perception at presentation to $20 / 200$ at 13 months' follow-up. The patient was treated with prompt PPV soon after presentation and was started on corticosteroids and ATT before culture results, based on the miliary lung disease discovered on CT scan. ${ }^{35}$ In the report by Yaghoubi et al, a $45-$ year-old man with concomitant TB pericarditis underwent a prompt tap-and-inject of vancomycin and amikacin. Oral corticosteroids were started early given the concomitant cardiac disease. Quadruple ATT could not be maintained for the total duration of treatment but isoniazid and ethambutol were given for 18 months. The visual acuity improvement from $20 / 630$ to $20 / 32$ was maintained for up to 3 years. $^{27}$

\section{Microbiologic Studies}

Table 8 summarizes the best reported method of diagnosis and microbiologic findings. A TB etiology was confirmed on histopathologic specimen (HPS) after removal of the eye in 32/44 cases $(72.7 \%)$. The earliest source of TB confirmation came from the vitreous (PPV or tap) in 6/44 cases $(13.6 \%)$ and via an AC tap in $3 / 44$ cases $(6.8 \%)$. The studies reported a variety of microbiologic studies including microscopy to look for acid-fast bacilli (AFB) and LGCs. Mycobacterial cultures, PCR and genomic deletion analysis were also used. Microscopy revealed AFB in 33/ 43 cases $(76.7 \%)$ and LGCs only in $7 / 43$ cases $(16.3 \%)$. Mycobacterial cultures from vitreous, anterior chamber or HPS were reported in $18 / 43$ cases $(41.9 \%)$ and were positive in $14 / 18$ cases $(77.8 \%)$. PCR was reported in 12 / 43 cases $(27.9 \%)$ and was positive in all cases. Detailed case-by-case descriptions are available in Table 3. The most specific reported microbiologic finding was M. tuberculosis in 19/44 cases (43.2\%), AFB in 17/44 cases $(38.6 \%)$, LGCs in $6 / 44$ cases $(13.6 \%)$ and $M$. bovis in $1 / 44$ case $(2.3 \%)$. One case from 2006 reported "TB etiology" as their final microbiologic finding. ${ }^{19}$

\section{Discussion}

Making the diagnosis of ocular TB is a challenge. There is a lack of comprehensive evidence on this topic and wide differences in institutional practices for the workup of patients. $^{38,39}$ In an effort to unify the diagnostic criteria for 
Table 7 Diagnostic Yield of Culture versus PCR for Mycobacterium tuberculosis Complex in Studies Where Both Tests Were Performed

\begin{tabular}{|c|c|c|c|c|}
\hline Author & $\#$ & $\begin{array}{l}\text { Ocular Specimen } \\
\text { Source }\end{array}$ & PCR & Culture \\
\hline Grosse et $\mathrm{al}^{33}$ & 16 & $\begin{array}{l}\text { Eyeball tissue from } \\
\text { enucleation }\end{array}$ & + & + \\
\hline Sen et $\mathrm{al}^{31}$ & 17 & $\begin{array}{l}\text { Eyeball tissue from } \\
\text { enucleation }\end{array}$ & + & - \\
\hline $\begin{array}{l}\text { Srichatrapimuk } \\
\text { et } \mathrm{al}^{21}\end{array}$ & 35 & $\begin{array}{l}\text { Vitreous aspirate from } \\
\text { vitreous tap }\end{array}$ & - & - \\
\hline \multirow[t]{11}{*}{ Rishi et $\mathrm{al}^{25}$} & \multirow[t]{4}{*}{38} & $\begin{array}{l}\text { Vitreous aspirate from } \\
\text { diagnostic vitrectomy }\end{array}$ & + & + \\
\hline & & $\begin{array}{l}\text { Vitreous aqueous } \\
\text { junction from diagnostic } \\
\text { vitrectomy }\end{array}$ & + & + \\
\hline & & $\begin{array}{l}\text { Mid-vitreous from } \\
\text { diagnostic vitrectomy }\end{array}$ & + & + \\
\hline & & $\begin{array}{l}\text { Organized tissue from } \\
\text { diagnostic vitrectomy }\end{array}$ & + & + \\
\hline & 39 & $\begin{array}{l}\text { Vitreous aspirate from } \\
\text { vitrectomy }\end{array}$ & + & + \\
\hline & \multirow[t]{2}{*}{40} & $\begin{array}{l}\text { Aqueous aspirate from } \\
\text { anterior chamber tap }\end{array}$ & + & - \\
\hline & & $\begin{array}{l}\text { Vitreous sample from } \\
\text { diagnostic vitrectomy }\end{array}$ & + & - \\
\hline & \multirow[t]{3}{*}{$4 I$} & $\begin{array}{l}\text { Aqueous aspirate from } \\
\text { vitrectomy }\end{array}$ & + & + \\
\hline & & $\begin{array}{l}\text { Lens aspirate from } \\
\text { vitrectomy }\end{array}$ & + & - \\
\hline & & $\begin{array}{l}\text { Vitreous aspirate from } \\
\text { vitrectomy }\end{array}$ & + & + \\
\hline & 42 & $\begin{array}{l}\text { Vitreous sample from } \\
\text { evisceration }\end{array}$ & + & - \\
\hline Antaki et $\mathrm{al}^{22}$ & 43 & $\begin{array}{l}\text { Diluted vitreous sample } \\
\text { from diagnostic } \\
\text { vitrectomy }\end{array}$ & + & + \\
\hline Yaghoubi et $\mathrm{al}^{27}$ & 44 & $\begin{array}{l}\text { Vitreous aspiration from } \\
\text { vitreous tap }\end{array}$ & + & - \\
\hline
\end{tabular}

Abbreviation: PCR, polymerase chain reaction.

TB uveitis, the Collaborative Ocular Tuberculosis Study (COTS-1) proposed in 2017 some diagnostic criteria based on a large multicentric retrospective study that included 1272 eyes. $^{39}$ Generally speaking, a comprehensive and
Table 8 Summary of the Best Reported Method of Diagnosis and Microbiologic Findings

\begin{tabular}{|l|l|l|}
\hline Characteristics & $\begin{array}{l}\text { Number } \\
\text { of } \\
\text { Patients }\end{array}$ & Percentage \\
\hline $\begin{array}{l}\text { Earliest source of TB } \\
\text { confirmation }\end{array}$ & & \\
Histopathologic specimen after & $32 / 44$ & $72.7 \%$ \\
removal of the eye & & \\
Vitreous (from vitrectomy or tap) & $6 / 44$ & $13.6 \%$ \\
AC tap & $3 / 44$ & $6.8 \%$ \\
Other (FNA, pus) & $3 / 44$ & $6.8 \%$ \\
\hline Microbiologic studies on the & & \\
earliest specimen allowing TB & & \\
confirmation* & & \\
Microscopy (reporting AFB) & $33 / 43$ & $76.7 \%$ \\
Microscopy (reporting LGC) & $7 / 43$ & $16.3 \%$ \\
Mycobacterial culture & $18 / 43$ & $41.9 \%$ \\
Positive & $14 / 18$ & $77.8 \%$ \\
$\quad$ Negative & $4 / 18$ & $22.2 \%$ \\
PCR & $12 / 43$ & $27.9 \%$ \\
Positive & $12 / 12$ & $100.0 \%$ \\
Genomic deletion analysis & $1 / 43$ & $2.3 \%$ \\
Not specified & $1 / 44$ & \\
\hline Best reported final microbiologic & & \\
findings & & \\
M. tuberculosis & $19 / 44$ & $43.2 \%$ \\
AFB & $17 / 44$ & $38.6 \%$ \\
LGC & $6 / 44$ & $13.6 \%$ \\
M. bovis & $1 / 44$ & $2.3 \%$ \\
"TB etiology" & $1 / 44$ & $2.3 \%$ \\
\hline
\end{tabular}

Note: *Categories not mutually exclusive.

Abbreviations: TB, tuberculosis/tuberculous; AC, anterior chamber; FNA, fineneedle aspiration; AFB, acid-fast bacilli; LGC, Langerhans giant cells.

multidisciplinary approach to ocular TB is favoured, including a thorough combination of history, examination, and screening investigations including TST/IGRA and CXR. In addition to ocular signs, the diagnosis can be supported by extraocular findings. ${ }^{38}$ The purpose of our study was to systematically review the available literature on endogenous TB endophthalmitis and panophthalmitis (severe subtypes of TB uveitis) in order to have a better understanding of the clinical features and evolution of the disease.

Our review shows that endogenous TB endophthalmitis and panophthalmitis can present in every age group, ranging from infants to the elderly. The disease most commonly presented in immunocompetent individuals but it is worth noting that $25.0 \%$ of patients were immunocompromised. Previous studies have linked intraocular tuberculosis to HIV. $^{8}$ In our cohort, concurrent HIV was seen in $16.7 \%$ of 
patients, with CD4 counts ranging from 34 to 263 cells $/ \mathrm{mm}^{3}$. Most patients $(63.2 \%)$ had no prior history of tuberculosis, making the ocular presentation the first manifestation of tuberculosis. Only $16.2 \%$ of patients had known active tuberculosis at presentation. Half of the patients did not report systemic symptoms like fever, chills or cough prior to presenting to the ophthalmology clinic. This highlights the importance of obtaining a thorough medical history from the patient, specially focused on risk factors such as the presence of immune disease, medication/drug use and atrisk behaviors. Even in the absence of systemic symptoms of tuberculosis or specific risk factors, a TB etiology must remain in the differential diagnosis of endogenous endophthalmitis and panophthalmitis.

In our review, $50.0 \%$ of the patients presented relatively late, 1 to 3 months after the beginning of symptoms. This could be due to a variety of reasons including difficulty with access to care and inappropriate medical management prior to referral to the eye specialist who made the diagnosis. Another possibility is that this disease leads to a slow deterioration of vision associated with gradually increasing symptoms. The ocular symptoms were the driving reason for consultation. Decreased vision was reported by $90.3 \%$ of patients, pain by $58.1 \%$ of patients and redness by $32.3 \%$ of patients. Abnormal protrusion of the eye was reported by $6.5 \%$ of patients. Visual acuity on presentation was very poor with $85.2 \%$ of patients presenting with $20 / 200$ or worse. Although bilateral presentation is frequent for ocular tuberculosis, there were only 4 reported cases of contralateral eye disease of lesser severity and no case of bilateral endophthalmitis or panophthalmitis. In parallel, follow-up durations were not rigorously reported in all studies. As such, we elected not to report them.

In more than half of the cases $(52.9 \%)$, the authors reported a presumptive diagnosis of endophthalmitis or panophthalmitis of unknown etiology, pending investigation. Before a definitive diagnosis was made, systemic antibiotics were prescribed in $47.6 \%$ of the reviewed cases. Corticosteroids alone were prescribed in $33.3 \%$ of cases without any antibiotic coverage. Current literature on endogenous endophthalmitis supports the initiation of treatment before a definitive diagnosis is made. Therapeutic approaches vary and the potential causative organism and extent of ocular involvement often direct the choice of therapy. Systemic antibiotics should have a wide spectrum of activity and very good ocular penetration. ${ }^{40}$ In parallel, corticosteroids modulate the host inflammatory response to infection and have been shown to reduce tuberculosis-associated mortality when given in conjunction with ATT. ${ }^{41}$ However, to our knowledge, there is no evidence to support the use of corticosteroids without ATT or antibiotic coverage. In our experience and based on current practice guidelines, if systemic corticosteroids were to be started in the context of endogenous endophthalmitis, this should be done in conjunction with antibiotics and/or antivirals. ${ }^{42}$

Retinoblastoma and other ocular malignancy were the presumptive diagnoses in $14.7 \%$ of cases. ${ }^{28,30-33}$ In the report by McMoli et al, a 1-year-old boy presented with orbital cellulitis and an episcleral mass on the left eye. Retinoblastoma was suspected and the patient received external radiotherapy. TB panophthalmitis was only diagnosed after removal of the eye and examination of the histopathologic specimen. This is a prime example of how intraocular TB can mimic other diseases, in this case causing a delay in the initiation of appropriate ATT and exposing the child to unnecessary ionizing radiation and enucleation. ${ }^{32}$ TB endophthalmitis can also mimic other infectious etiologies such as viral herpetic disease and toxoplasmosis, presenting as ARN. In the cases described by Antaki et al and by Rishi et al, peripheral retinal necrosis was seen along with panuveitis, prompting a diagnosis of ARN. In both cases, PCR on the vitreous samples confirmed the TB etiology. ${ }^{22,25}$

Common investigations for endogenous endophthalmitis include CXR, CT scan, TST and/or IGRA. Abnormal chest imaging was seen in $70.4 \%$ of cases and this included any abnormal finding reported by the authors (miliary pattern, "lesion", "active infection", consolidation, etc.). In 3 cases, despite a normal initial CXR, further imaging with CT scan revealed a miliary pattern. A CXR alone is not sensitive enough to rule out pulmonary tuberculosis. If resources permit, CT scan of the chest should be obtained in all cases as it permits the detection of lesions even in cases with normal radiography. ${ }^{43}$ A TST was positive in the majority of cases $(60.0 \%)$ overall and in $4 / 9$ (44.4\%) of the immunocompromised individuals who underwent skin testing. However, a negative TST cannot rule out a TB etiology. A main cause for a false negative TST is anergy secondary to immunosuppression or disseminated tuberculosis. ${ }^{44}$ Repeat TST might be useful since conversion can occur along the disease course. IGRA testing was not widely reported possibly due to the lack of this technology at the time and place of publication in many the studied cases. Among the 4 cases where it was 
reported, one case showed a negative TST with a positive IGRA. $^{25}$

Diagnostic interventions were performed prior to the final outcome or removal of the eye in half of the patients. PPV was performed in $23.5 \%$ of cases and a vitreous tap was obtained alone or before PPV in $20.6 \%$ of cases. This finding is consistent with a large meta-analysis of endogenous endophthalmitis cases reported between 1986 and 2012 that revealed a $32 \%$ rate of PPV in that series. ${ }^{45}$ It is important to note that the frequency and usefulness of PPV might be underestimated in our series since PPV is a relatively modern technique and access to a vitrectomy machine might not be readily available in every context. Regarding microbiological yield, vitreous specimens obtained during PPV had the highest yield with $87.5 \%$ of specimens returning positive for mycobacteria. Undiluted vitreous samples from vitreous taps were positive in only $14.3 \%$ of cases. Theoretically, since a greater sample of vitreous is obtained during PPV, the yield of PPV should be higher than the yield of vitreous tap. Consistent with our data, previous studies have shown that culturing contents of the vitrectomy cassette increases the likelihood of obtaining a positive culture compared to vitreous biopsy. ${ }^{46}$ However, our data contrasts with the results of the Endophthalmitis Vitrectomy Study (EVS) that examined cases of post-operative bacterial endophthalmitis. In the EVS, PPV did not produce significantly more positive cultures than tap and the authors recommended against performing PPV solely to improve microbiological yield. ${ }^{47}$ Possible explanations include differences in causative organisms, variations in PPV and vitreous tap techniques, as well as disparities in microbiologic analysis methods. Regarding the yield of mycobacterial PCR versus culture, our study shows that the sensitivity of cultures was not high enough $(60.0 \%)$ to allow clinicians to rely on negative cultures for ruling out an intraocular TB infection. PCR appears to be more accurate than cultures in diagnosing intraocular TB. This is in keeping with previous studies showing high PCR positivity in cases of clinically suspected ocular TB. ${ }^{48,49}$ However, this result needs to be interpreted with caution given the small sample size in our study. The COTS-1 Report 3 studied the real-world evidence on the utility of PCR of ocular fluids in the management of ocular TB. ${ }^{50}$ It showed that PCR analysis still needs significant advancement to gain wider acceptability worldwide. ${ }^{50}$ The report also demonstrated that PCR analysis may not influence management or treatment outcomes in the real-world scenario. ${ }^{50}$ Regarding cost-effectiveness, previous studies looking at the diagnosis of pulmonary TB have demonstrated significant cost saving benefits of PCR versus mycobacterial culture. ${ }^{51}$ From our study, no extrapolations can be made about the cost-effectiveness of those techniques for ocular fluid analysis. However, at our institution, the costs associated with mycobacterial culture of a specimen in the setting of suspicion of TB are 85.20 Canadian dollars, whereas the cost of direct PCR for mycobacteria is 72.00 Canadian dollars. $^{52}$

Systemic disease was frequently present in the reviewed cases. Pulmonary tuberculosis was reported in $42.8 \%$ of cases and miliary tuberculosis in $20.0 \%$ of cases Other extraocular manifestations were reported but were not labeled as miliary tuberculosis. Those included: CNS, abdominal and peritoneal, pericardial and adrenal. ${ }^{19,21,27,28,31,37,53}$ Treatment regimens varied from case-to-case and included medications like rifampin, isoniazid, pyrazinamide and ethambutol, among others. The reported mortality in this series was low (1 case) but this could be due to under-reporting as follow-up duration varied significantly from study to study. Rapid consultation with an infectious disease specialist should be made early to investigate systemic findings and manage the treatment. $^{22}$ Caution should be exercised with ATT since isoniazid and ethambutol can both cause toxic optic neuropathies. $^{54}$ Those toxicities are usually dosedependent and associated with prolonged used. ${ }^{9}$ Despite potential toxicity, those agents often cannot be stopped given the high mortality associated with disseminated infection and the paucity of efficient antituberculous agents in the case of CNS disease. ${ }^{22}$

Ocular outcomes were generally very poor with $83.7 \%$ of cases requiring either enucleation, evisceration or exenteration. This is significantly higher than the reported rate of enucleation in cases of endogenous bacterial endophthalmitis, as demonstrated in a large study of 2014 which reports a $20 \%$ enucleation rate. ${ }^{45}$ This highlights the significant challenge associated with the diagnosis of TB endophthalmitis and panophthalmitis. This disease has no pathognomonic eye findings and can occur in apparently healthy individuals. Diagnosis is often delayed which can lead to profound visual and organ loss. ${ }^{38,55}$ Starting ATT as early as possible might be beneficial to decrease vision loss and to prevent mortality. However, the decision to start empiric treatment in the absence of laboratory-based diagnosis remains difficult given the known toxicity associated with these 
medications. PPV has been shown to be beneficial in preserving vision and decreasing the likelihood of progression to enucleation in cases of endogenous bacterial endophthalmitis. ${ }^{45}$ PPV might have the same benefits in cases of a TB etiology by providing an earlier diagnosis (highest diagnostic yield) and decreasing the infectious and inflammatory intraocular load.

Our study has several limitations. First, the data were gathered only from case reports and case series which are known to have selection and publication bias. We report poor ocular outcomes associated with endogenous TB endophthalmitis and panophthalmitis, but we recognize that severe cases are more likely to be reported by authors. We performed a quantitative analysis of the extracted data but our results could not produce relative association measures. This is an intrinsic limitation of the methodology of systematic reviews of case reports and case series, due to the heterogeneity of the data. To counter missing data and to study the highest numbers of clinical characteristics, we performed available-case analysis. The total number of observations varied between parameters and, in some cases, the sample sizes were small. As such, some of the results must be interpreted with caution. While not all the included reports relied on a molecular diagnosis of MTBC, all efforts were made to only incorporate studies describing cases compatible with hematogenous spread of tuberculosis. Our analysis captured the heterogeneity of the management of TB endophthalmitis and panophthalmitis which is affected by the availability of technologies and resources at the time of publication (eg, IGRA, CT scan and PPV). Despite these limitations, we believe that our results can be useful to ophthalmologists as they can get a better appreciation of this rare condition. To our knowledge, this is the largest comprehensive review of endogenous TB endophthalmitis and panophthalmitis.

\section{Conclusion}

We can make the following conclusions and recommendations based on the results of this study as well as our clinical experience. In patients presenting with endogenous endophthalmitis and panophthalmitis of unknown etiology: (1) maintaining a high index of suspicion for tuberculosis is required to provide timely diagnosis and management (evidence: study results); (2) a thorough medical history should be obtained with a focus on prior history of tuberculosis, immunosuppression and medication use (evidence: study results); (3) CT scan of the chest should be considered even in cases with a normal initial CXR, and orbital/cerebral imaging should be considered as well (evidence: study results); (4) mycobacterial cultures and PCR should be done on every vitreous specimen obtained in the context of an infection if the vitreal sample volume permits and especially in unusual cases and in patients coming from endemic areas (evidence: clinical experience). In patients with confirmed TB endophthalmitis/panophthalmitis, we recommend: (1) prompt consultation with an infectious disease specialist to investigate for systemic disease even in the absence of generalized symptoms (evidence: study results); (2) testing for HIV after discussion with the patient since tuberculosis and HIV frequently occur together (evidence: study results); (3) after the initiation of ATT, monitoring for potential medication-induced optic neuropathy (evidence: clinical experience); and (4) given the grim ocular prognosis and to strengthen the patient-doctor therapeutic alliance, patients should be counselled early on the high likelihood of permanent vision and organ loss despite maximal therapy (evidence: clinical experience and study results).

\section{Author Contributions}

All authors made substantial contributions to conception and design, acquisition of data, or analysis and interpretation of data; took part in drafting the article or revising it critically for important intellectual content; agreed to submit to the current journal; gave final approval of the version to be published; and agree to be accountable for all aspects of the work.

\section{Funding}

There is no funding to report.

\section{Disclosure}

The authors declare that they have no conflicts of interest for this work.

\section{References}

1. Raviglione M, Sulis G. Tuberculosis 2015: burden, challenges and strategy for control and elimination. Infect Dis Rep. 2016;8(2):6570.

2. World Health Organization. Global tuberculosis report 2015. 2015.

3. Centers for Disease Control and Prevention. Trends in tuberculosis, 2018. Published 2019. Available from: https://www.cdc.gov/tb/publica tions/factsheets/statistics/tbtrends.htm. Accessed April 27, 2020.

4. Cole ST, Brosch R, Parkhill J, et al. Deciphering the biology of mycobacterium tuberculosis from the complete genome sequence. Nature. 1998;393(6685):537-544.

5. Scott C, Cavanaugh JS, Pratt R, Silk BJ, LoBue P, Moonan PK. Human tuberculosis caused by mycobacterium bovis in the United States, 2006-2013. Clin Infect Dis. 2016;63(5):594-601.

6. Bobadilla-del Valle M, Torres-Gonzalez P, Cervera-Hernandez ME, et al. Trends of mycobacterium bovis isolation and first-line anti-tuberculosis drug susceptibility profile: a fifteen-year laboratory-based surveillance. PLoS Negl Trop Dis. 2015;9(9):e0004124. 
7. Hernandez-Solis A, Gonzalez-Villa M, Cicero-Sabido R, et al. Identificacion de Mycobacterium bovis en pacientes con diagnostico de tuberculosis pulmonar y extrapulmonar. Gac Med Mex. 2019;155 (6):608-612.

8. Sanches I, Carvalho A, Duarte R. Who are the patients with extrapulmonary tuberculosis? Rev Port Pneumol. 2015;21(2):90-93.

9. Dalvin LA, Smith WM. Intraocular manifestations of mycobacterium tuberculosis: a review of the literature. J Clin Tuberculosis Other Mycobacterial Dis. 2017;7:13-21.

10. Gupta V, Gupta A, Arora S, Bambery P, Dogra MR, Agarwal A. Presumed tubercular serpiginouslike choroiditis: clinical presentations and management. Ophthalmology. 2003;110(9):1744-1749.

11. Gupta V, Gupta A, Rao NA. Intraocular tuberculosis - an update. Surv Ophthalmol. 2007;52(6):561-587.

12. Khochtali S, Gargouri S, Abroug N, et al. The spectrum of presumed tubercular uveitis in Tunisia, North Africa. Int Ophthalmol. 2015;35 (5):663-671.

13. Sharma A, Thapa B, Lavaju P. Ocular tuberculosis: an update. Nepal J Ophthalmol. 2011;3(1):52-67.

14. Gan WL, Jones NP. Serpiginous-like choroiditis as a marker for tuberculosis in a non-endemic area. Br J Ophthalmol. 2013;97 (5):644-647.

15. Tejada P, Mendez MJ, Negreira S. Choroidal tubercles with tuberculous meningitis. Int Ophthalmol. 1994;18(2):115-118.

16. Moher D, Liberati A, Tetzlaff J, Altman DG, Group P. Preferred reporting items for systematic reviews and meta-analyses: the PRISMA statement. BMJ. 2009;339:b2535.

17. Peugh JL, Enders CK. Missing data in educational research: a review of reporting practices and suggestions for improvement. Rev Educ Res. 2004;74(4):525-556.

18. Menezo JL, Martinez-Costa R, Marin F, Vilanova E, CortesVizcaino V. Tuberculous panophthalmitis associated with drug abuse. Int Ophthalmol. 1987;10(4):235-240.

19. Babu RB, Sudharshan S, Kumarasamy N, Therese L, Biswas J. Ocular tuberculosis in acquired immunodeficiency syndrome. Am J Ophthalmol. 2006;142(3):413-418.

20. Boonsopon S, Tesavibul N, Uiprasertkul M, Leeamornsiri S, Choopong P. Rare presentation of intractable tuberculous panophthalmitis with intraocular and intraorbital abscesses: a case report. $J$ Med Case Rep. 2017;11(1):180.

21. Srichatrapimuk S, Wattanatranon D, Sungkanuparph S. Tuberculous panophthalmitis with lymphadenitis and central nervous system tuberculoma. Case Rep Infect Dis. 2016;2016:6785382.

22. Antaki F, Marchand-Senecal X, Aubin MJ. Endogenous endophthalmitis and bilateral optic neuropathy secondary to Mycobacterium bovis. Can J Ophthalmol. 2020;55(3):e88-e91.

23. Biswas J, Madhavan HN, Gopal L, Badrinath SS. Intraocular tuberculosis. Clinicopathologic study of five cases. Retina. 1995;15 (6):461-468.

24. Anders N, Wollensak G. Ocular tuberculosis in systemic lupus erythematosus and immunosuppressive therapy. Klin Monbl Augenheilkd. 1995;207(6):368-371.

25. Rishi E, Rishi P, Therese KL, et al. Culture and reverse transcriptase polymerase chain reaction (RT-PCR) proven mycobacterium tuberculosis endophthalmitis: a case series. Ocul Immunol Inflamm. 2018;26 (2):220-227.

26. Sengupta S, Tomar VP, Biswas J. Coexistent miliary tuberculosis of choroid and tubercular panuveitis: a report. Indian J Ophthalmol. 2013;61(12):761-762.

27. Yaghoubi GH, Abedi F, Ziaee M, Norouzpour A. Tuberculosis: a cunning disease presenting with endopericarditis-associated bilateral uveitis. Turk J Ophthalmol. 2019;49(6):361-363.

28. Ni C, Papale JJ, Robinson NL, Wu BF. Uveal tuberculosis. Int Ophthalmol Clin. 1982;22(3):103-124.

29. Regillo CD, Shields CL, Shields JA, Eagle RC, Lehr J. Ocular tuberculosis. JAMA. 1991;266(11):1490.
30. Raina UK, Tuli D, Arora R, Mehta DK, Taneja M. Tubercular endophthalmitis simulating retinoblastoma. Am J Ophthalmol. 2000;130(6):843-845.

31. Sen S, Kashyap S, Singh UB, NagaSuresh V, Chand M, Garg SP. Intraocular tuberculosis mimicking retinoblastoma: a case report. Diagn Cytopathol. 2003;28(2):107-109.

32. McMoli TE, Mordi VP, Grange A, Abiose A. Tuberculous panophthalmitis. J Pediatr Ophthalmol Strabismus. 1978;15(6):383-385.

33. Grosse V, Bange FC, Tischendorf J, Schmidt RE, Manns MP. A mass in the eye. Lancet. 2002;360(9337):922.

34. Wadhwani M, Sethi S, Beri S, et al. An unusual case of metastatic tubercular panophthalmitis in a 14-year-old boy. $J$ Pediatr Ophthalmol Strabismus. 2011;48(5):318-319.

35. Hase K, Namba K, Saito W, Ohno S, Ishida S. A case of tuberculous endophthalmitis successfully treated with vitrectomy followed by antituberculous agents. J Ophthalmic Inflamm Infect. 2015;5:14.

36. Darrell RW. Acute tuberculous panophthalmitis. Arch Ophthalmol. 1967;78(1):51-54.

37. Dvorak-Theobald G. Acute tuberculous endophthalmitis; report of a case. Am J Ophthalmol. 1958;45(3):403-407.

38. Patel SS, Saraiya NV, Tessler HH, Goldstein DA. Mycobacterial ocular inflammation: delay in diagnosis and other factors impacting morbidity. JAMA Ophthalmol. 2013;131(6):752-758.

39. Agrawal R, Gunasekeran DV, Grant R, et al. Clinical features and outcomes of patients with tubercular uveitis treated with antitubercular therapy in the collaborative ocular tuberculosis study (COTS)-1. JAMA Ophthalmol. 2017;135(12):1318-1327.

40. Birnbaum F, Gupta G. Endogenous endophthalmitis: diagnosis and treatment. Published 2016. Available from: https:/www.aao.org/eye net/article/endogenous-endophthalmitis-diagnosis-treatment. Accessed May 4, 2020.

41. Critchley JA, Young F, Orton L, Garner P. Corticosteroids for prevention of mortality in people with tuberculosis: a systematic review and meta-analysis. Lancet Infect Dis. 2013;13(3):223-237.

42. American Academy of Ophthalmology. Practicing Ophthalmologists Curriculum Uveitis. 2014

43. Alkabab YM, Enani MA, Indarkiri NY, Heysell SK. Performance of computed tomography versus chest radiography in patients with pulmonary tuberculosis with and without diabetes at a tertiary hospital in Riyadh, Saudi Arabia. Infect Drug Resist. 2018;11:37-43.

44. Nayak S, Acharjya B. Mantoux test and its interpretation. Indian Dermatol Online J. 2012;3(1):2-6.

45. Jackson TL, Paraskevopoulos T, Georgalas I. Systematic review of 342 cases of endogenous bacterial endophthalmitis. Surv Ophthalmol. 2014;59(6):627-635.

46. Donahue SP, Kowalski RP, Jewart BH, Friberg TR. Vitreous cultures in suspected endophthalmitis. Biopsy or vitrectomy? Ophthalmology. 1993;100(4):452-455.

47. Barza M, Pavan PR, Doft BH, et al. Evaluation of microbiological diagnostic techniques in postoperative endophthalmitis in the endophthalmitis vitrectomy study. Arch Ophthalmol. 1997;115 (9):1142-1150.

48. Balne PK, Modi RR, Choudhury N, et al. Factors influencing polymerase chain reaction outcomes in patients with clinically suspected ocular tuberculosis. J Ophthalmic Inflamm Infect. 2014;4(1):10.

49. Biswas J, Kazi MS, Agarwal VA, Alam MS, Therese KL. Polymerase chain reaction for mycobacterium tuberculosis DNA detection from ocular fluids in patients with various types of choroiditis in a referral eye center in India. Indian J Ophthalmol. 2016;64(12):904-907.

50. Agarwal A, Agrawal R, Gunasekaran DV, et al. The collaborative ocular tuberculosis study (COTS)-1 report 3: polymerase chain reaction in the diagnosis and management of tubercular uveitis: global trends. Ocul Immunol Inflamm. 2019;27(3):465-473.

51. Scherer LC, Sperhacke RD, Ruffino-Netto A, et al. Costeffectiveness analysis of PCR for the rapid diagnosis of pulmonary tuberculosis. BMC Infect Dis. 2009;9:216. 
52. Ministère de la Santé et des Services sociaux. Répertoire québécois et système de mesure des procédures de biologie médicale. 2019.

53. Demirci H, Shields CL, Shields JA, Eagle RC. Ocular tuberculosis masquerading as ocular tumors. Surv Ophthalmol. 2004;49(1):78-89.

54. Jimenez-Lucho VE, Del Busto R, Odel J. Isoniazid and ethambutol as a cause of optic neuropathy. Eur J Respir Dis. 1987;71(1):42-45.

55. Vaziri K, Schwartz SG, Kishor K, Flynn HW. Endophthalmitis: state of the art. Clin Ophthalmol. 2015;9:95-108.

56. Manthey KF, Duncker G, Gronemeyer U. [Endophthalmitis caused by mycobacterium tuberculosis]. Klin Monbl Augenheilkd. 1982;180 (6):556-558.

57. Sheu SJ, Shyu JS, Chen LM, Chen YY, Chirn SC, Wang JS. Ocular manifestations of tuberculosis. Ophthalmology. 2001;108 (9):1580-1585.
58. Chawla R, Garg S, Venkatesh P, Kashyap S, Tewari HK. Case report of tuberculous panophthalmitis. Med Sci Monit. 2004;10(10):Cs5759.

59. Wroblewski KJ, Hidayat AA, Neafie RC, Rao NA, Zapor M. Ocular tuberculosis: a clinicopathologic and molecular study. Ophthalmology. 2011;118(4):772-777.

60. Agarwal S, Gupta P, Pandey P, Ralli M. Tubercular panophthalmitis: case report of a rare entity. Med J Dr DY Patil Univ. 2017;10 (4):390-392.

\section{Publish your work in this journal}

Clinical Ophthalmology is an international, peer-reviewed journal covering all subspecialties within ophthalmology. Key topics include: Optometry; Visual science; Pharmacology and drug therapy in eye diseases; Basic Sciences; Primary and Secondary eye care; Patient Safety and Quality of Care Improvements. This journal is indexed on PubMed

Submit your manuscript here: https://www.dovepress.com/clinical-ophthalmology-journal
Central and CAS, and is the official journal of The Society of Clinical Ophthalmology (SCO). The manuscript management system is completely online and includes a very quick and fair peer-review system, which is all easy to use. Visit http://www.dovepress.com/ testimonials.php to read real quotes from published authors. 